\title{
Ray class fields of global function fields with many rational places
}

\author{
by
}

\author{
Roland AuER (Groningen)
}

0. Introduction. Algebraic curves over finite fields with many rational points have been of increasing interest in the last two decades. The question of explicitly determining the maximal number of points on a curve of given genus was initiated and in some special cases solved by Serre $[34,35,36]$ around 1982. Since then there have been attempts to attack the problem by means of algebraic geometry as well as field arithmetic. Constructions by explicit equations have been carried out by van der Geer and van der Vlugt $[7,8]$. The present paper, which makes use of class field theory, has its immediate predecessors in work by Lauter [13, 14, 15] and Niederreiter and Xing [19, 20, 21, 25, 26, 38]. The numerical results obtained improve several entries of the tables given in [9], [17] and [27].

As we are looking from the field theoretic point of view, with an algebraic curve $X$ (smooth, projective, absolutely irreducible) defined over a finite field $\mathbb{F}_{q}$ we associate its field $K=\mathbb{F}_{q}(X)$ of algebraic functions, a global function field with full constant field $\mathbb{F}_{q}$. Its genus is that of $X$, and coverings of $X$ correspond to field extensions of $K$, the degree of the covering being the degree of the extension.

A place of $K$, by which we mean the maximal ideal $\mathfrak{p}$ in some discrete valuation ring of $K$, with (residue field) degree $d=\operatorname{deg} \mathfrak{p}$, corresponds to (a Galois conjugacy class of $) d$ points on $X\left(\mathbb{F}_{q^{d}}\right)$, and each point on $X$ having $\mathbb{F}_{q^{d}}$ as its minimal field of definition over $\mathbb{F}_{q}$ lies in such a conjugacy class. In particular the rational places, i.e. the places of degree 1 , of $K \mid \mathbb{F}_{q}$ are in 1-1 correspondence with the $\mathbb{F}_{q}$-rational points on $X$. The (normalized) discrete valuation associated with a place $\mathfrak{p}$ of $K$ will be denoted by $v_{\mathfrak{p}}$.

2000 Mathematics Subject Classification: Primary 11G20; Secondary 11R37, 11R58, 14G15, 11Y40, 11R29.

Key words and phrases: ray class fields, global function fields, characteristic $p$, curves with many rational points, $S$-class numbers. 
Our approach to the problem of constructing curves with many rational points will be by ray class fields: With each non-empty set $S$ of places and each effective divisor $\mathfrak{m}$ of $K$ with support disjoint from $S$ we associate a global function field $K_{S}^{\mathfrak{m}}$, the largest abelian extension of $K$ with conductor $\leq \mathfrak{m}$ in which every place of $S$ splits completely. These ray class fields have been used by Perret [30] before, but because of some misleading statements in his paper, we shall give a complete proof of their existence and derive some of their basic properties, a task which is performed in Section 1.

Section 2 discusses upper bounds for $S$-class numbers and for the number of rational places of a global function field. In Section 3 we prove the existence of curves with many rational points by explicitly computing the needed invariants of certain $K_{S}^{\mathfrak{m}}$. At this point I want to thank the referee for drawing my attention to recent articles by Niederreiter and Xing, which have appeared after submission of this paper and could be added to the references during the revision.

1. Ray class fields. Let $K$ be a global function field with full constant field $\mathbb{F}_{q}$. Denote by $\mathcal{I}=\mathcal{I}_{K}$ and $\mathcal{C}=\mathcal{C}_{K}=\mathcal{I} / K^{*}$ the idèle group and the idèle class group of $K$, respectively. (The diagonal embedding of $K^{*}$ into $\mathcal{I}$ is considered as inclusion.)

Given a place $\mathfrak{p}$ of $K$, let $K_{\mathfrak{p}}$ be the completion of $K$ at $\mathfrak{p}$, and $\overline{\mathfrak{p}}$ the extension of $\mathfrak{p}$ to $K_{\mathfrak{p}}$ (i.e. the topological closure of $\mathfrak{p}$ in $K_{\mathfrak{p}}$ ). Furthermore we have the $n$th one-unit group $U_{\mathfrak{p}}^{(n)}=1+\overline{\mathfrak{p}}^{n}$, where we also admit $n=0$, in which case we mean the usual unit group $U_{\mathfrak{p}}=\left\{u \in K_{\mathfrak{p}}^{*} \mid v_{\overline{\mathfrak{p}}}(u)=0\right\}$. The canonical embedding of $K_{\mathfrak{p}}^{*}$ into $\mathcal{I}$ will be denoted by [ $]_{\mathfrak{p}}$, i.e. $[z]_{\mathfrak{p}}$ for $z \in K_{\mathfrak{p}}^{*}$ is the idèle $(\ldots, 1, z, 1, \ldots)$ having $z$ at its pth position and 1 elsewhere.

Let $S$ be a set of places of $K$. By an $S$-cycle (of $K$ ) we mean an effective divisor of $K$ with support disjoint from $S$. Let $\mathfrak{m}=\sum m_{\mathfrak{p}} \mathfrak{p}$ be such an $S$-cycle. Then, following Perret [30, pp. 305ff], we define the $S$-congruence subgroups $\bmod \mathfrak{m}$,

$$
\mathcal{I}_{S}^{\mathfrak{m}}:=\left(\prod_{\mathfrak{p} \in S} K_{\mathfrak{p}}^{*} \times \prod_{\mathfrak{p} \notin S} U_{\mathfrak{p}}^{\left(m_{\mathfrak{p}}\right)}\right) \cap \mathcal{I} \quad \text { and } \quad \mathcal{C}_{S}^{\mathfrak{m}}:=K^{*} \mathcal{I}_{S}^{\mathfrak{m}} / K^{*}
$$

of $\mathcal{I}$ and $\mathcal{C}$, respectively. (Here intersection with $\mathcal{I}$ is only necessary, of course, when $S$ is infinite.) We also put $\mathcal{I}^{\mathfrak{m}}:=\mathcal{I}_{S(\mathfrak{m})}^{\mathfrak{m}}$ where $S(\mathfrak{m})$ denotes the set of all places of $K$ not occurring in the support of $\mathfrak{m}$.

For the purposes of class field theory, $\mathcal{I}$ carries the restricted product topology of the $K_{\mathfrak{p}}^{*}$ with respect to the $U_{\mathfrak{p}}$ (cf. [2, p. 68]), which amounts to saying that $\mathcal{I}$ is a topological group and the congruence subgroups $\mathcal{I}_{\emptyset}^{\mathfrak{m}}$, where $\mathfrak{m}$ runs through all effective divisors of $K$, form a basis of open neighbourhoods of 1 . By definition the $\mathcal{I}_{S}^{\mathfrak{m}}$ are open (and thereby closed) subgroups of 
$\mathcal{I}$, and since $\mathcal{C}$ is endowed with the quotient topology induced from $\mathcal{I}$, the $\mathcal{C}_{S}^{\mathfrak{m}}$ are also open (and closed).

For any place $\mathfrak{p}$ of $K$ the kernel of the map $K_{\mathfrak{p}}^{*} \rightarrow \mathcal{I} / K^{*} \mathcal{I}_{\emptyset}^{\mathfrak{m}}$ induced by the embedding []$_{\mathfrak{p}}$ is contained in $\mathbb{F}_{q}^{*} U_{\mathfrak{p}}^{\left(m_{\mathfrak{p}}\right)} \subseteq U_{\mathfrak{p}}$. But $K_{\mathfrak{p}}^{*} / U_{\mathfrak{p}} \simeq \mathbb{Z}$, hence the index of $\mathcal{C}_{\emptyset}^{\mathfrak{m}}$ in $\mathcal{C}$ turns out to be infinite, contrary to the relevant assertions in [30, p. 302], which are therefore wrong. Due to this fact we assume $S$ non-empty from now on.

Denote by $\mathcal{O}_{S}$ the ring of all functions in $K$ with poles only in $S$, which is known to be a Dedekind domain with quotient field $K$. As is easily verified by means of (weak) approximation, the $S$-ray class group $\bmod \mathfrak{m}, \mathcal{C} / \mathcal{C}_{S}^{\mathfrak{m}} \simeq$ $\mathcal{I} / K^{*} \mathcal{I}_{S}^{\mathfrak{m}}$, is naturally isomorphic to the $S$-ideal class group $\bmod \mathfrak{m}, \mathcal{C l}^{\mathfrak{m}}\left(\mathcal{O}_{S}\right)$, defined as the quotient of the group of fractional ideals of $\mathcal{O}_{S}$ prime to $\mathfrak{m}$ by its subgroup of principal ideals $x \mathcal{O}_{S}$ with $x \in K^{*} \cap \mathcal{I}^{\mathfrak{m}}$. Note that for $\mathfrak{m}=\mathfrak{o}$, the zero divisor of $K$, we recover the usual ideal class group $\mathcal{C}\left(\mathcal{O}_{S}\right)$, whose cardinality, the $S$-class number (of $K$ ), is denoted by $h_{S}$.

\subsection{Proposition. The sequence}

$$
\mathcal{O}_{S}^{*} \rightarrow \mathcal{I}_{S}^{\mathfrak{o}} / \mathcal{I}_{S}^{\mathfrak{m}} \rightarrow \mathcal{I} / K^{*} \mathcal{I}_{S}^{\mathfrak{m}} \rightarrow \mathcal{I} / K^{*} \mathcal{I}_{S}^{\mathfrak{o}} \rightarrow 1
$$

is exact, and $\left(\mathcal{C}: \mathcal{C}_{S}^{\mathfrak{m}}\right)$ is finite.

Pr o of. We have $\mathcal{O}_{S}^{*}=K^{*} \cap \mathcal{I}_{S}^{\mathfrak{o}}$, implying $\mathcal{O}_{S}^{*} \mathcal{I}_{S}^{\mathfrak{m}} / \mathcal{I}_{S}^{\mathfrak{m}}=K^{*} \mathcal{I}_{S}^{\mathfrak{m}} \cap \mathcal{I}_{S}^{\mathfrak{o}} / \mathcal{I}_{S}^{\mathfrak{m}}$, which is the exactness at $\mathcal{I}_{S}^{\mathfrak{o}} / \mathcal{I}_{S}^{\mathfrak{m}}$. The exactness at the other groups is even simpler. As for the second claim note that both $\mathcal{I}_{S}^{\mathfrak{o}} / \mathcal{I}_{S}^{\mathfrak{m}} \simeq \prod_{\mathfrak{p}} U_{\mathfrak{p}} / U_{\mathfrak{p}}^{\left(m_{\mathfrak{p}}\right)}$ and $\mathcal{I} / K^{*} \mathcal{I}_{S}^{\mathfrak{o}} \simeq \mathcal{C} \ell\left(\mathcal{O}_{S}\right)$ are finite.

Let us briefly recall the essential facts of (global) class field theory (see $[2$, p. 172$]$ and $[16$, p. 408$])$ : For each finite Galois extension $L \mid K$ there is a surjective homomorphism

$$
(, L \mid K): \mathcal{C}_{K} \rightarrow G(L \mid K)^{\mathrm{ab}}
$$

from the idèle class group of $K$ onto the abelianization of the Galois group of $L \mid K$, called the norm residue symbol of $L \mid K$, which at the same time we think of as defined on $\mathcal{I}_{K}$. Its kernel is the norm group $\mathcal{N}_{L}:=N_{L \mid K} \mathcal{C}_{L}=$ $K^{*} N_{L \mid K} \mathcal{I}_{L} / K^{*}$, where $N_{L \mid K}$ denotes the norm from $L$ to $K$. Furthermore, the map $L \mapsto \mathcal{N}_{L}$ is an inclusion-reversing 1-1 correspondence between the finite abelian extensions (within some fixed algebraic closure $\bar{K}$ ) of $K$ and the closed subgroups of finite index in $\mathcal{C}_{K}$.

Accordingly we can define the $S$-ray class field $\bmod \mathfrak{m}$, denoted by $K_{S}^{\mathfrak{m}}$, as being the unique (in $\bar{K}$ ) abelian extension $L \mid K$ satisfying $\mathcal{N}_{L}=\mathcal{C}_{S}^{\mathfrak{m}}$. Then $K_{S}^{\mathfrak{o}}$ is just the Hilbert class field of $\mathcal{O}_{S}$ in the sense of Rosen [32] (with the slight generalization that we allow $S$ to be infinite). Moreover, in case $S$ consists of exactly one place, an explicit construction of $K_{S}^{\mathfrak{m}}$ via rank 1 Drinfel'd modules has been carried out by Hayes [11]. 
Next we want to investigate some extremality properties of the $S$-ray class fields. To this end we introduce the conductor of a finite abelian extension $L \mid K$ as the effective divisor

$$
\mathfrak{f}(L \mid K):=\sum_{\mathfrak{p}} f(L, \mathfrak{p}) \mathfrak{p}
$$

of $K$, with the conductor exponent $f(L, \mathfrak{p})$ of $\mathfrak{p}$ in $L$ being the least integer $n \geq 0$ such that the $n$th upper ramification group $G^{n}(L, \mathfrak{p})$ of $\mathfrak{p}$ in $L$ gets trivial. For a definition of the upper (index) ramification groups we refer the reader to [2, pp. 33ff], [16, pp. 186ff] or the appendix of this paper. By introducing the conductor in this way we avoid dealing with infinite class field extensions as encountered in Perret's paper [30]. For the following lemma, proposition and theorem cf. Section I of that paper. Because $G^{0}(L, \mathfrak{p})$ is the inertia group of $\mathfrak{p}$ in $L$, exactly the places occurring in $\mathfrak{f}(L \mid K)$ are ramified in $L$.

1.2. Conductor Lemma. Let $L \mid K$ be finite abelian and let $S$ be any non-empty set of places of $K$ splitting completely in $L$. Then $\mathfrak{f}(L \mid K)$ is the smallest $S$-cycle $\mathfrak{m}$ such that $L$ is contained in $K_{S}^{\mathfrak{m}}$ (i.e. $\mathcal{N}_{L}$ contains $\left.\mathcal{C}_{S}^{\mathfrak{m}}\right)$.

Proof. For any place $\mathfrak{p}$ of $K$ the composite map $K_{\mathfrak{p}}^{*} \hookrightarrow \mathcal{I} \rightarrow G(L \mid K)$ of the embedding []$_{\mathfrak{p}}$ with the norm residue symbol $(, L \mid K)$ has the decomposition group $G^{-1}(L, \mathfrak{p})$ as its image and maps $U_{\mathfrak{p}}^{(n)}$ onto $G^{n}(L, \mathfrak{p})$ for all $n \in \mathbb{N}_{0}$ (cf. [2, p. 155]). Hence we have

$$
\left[U_{\mathfrak{p}}^{(n)}\right]_{\mathfrak{p}} \subseteq K^{*} N_{L \mid K} \mathcal{I}_{L} \quad \Leftrightarrow \quad n \geq f(L, \mathfrak{p})
$$

and even $\left[K_{\mathfrak{p}}^{*}\right]_{\mathfrak{p}} \subseteq K^{*} N_{L \mid K} \mathcal{I}_{L}$ when $\mathfrak{p} \in S$.

Now let $\mathfrak{m}=\sum m_{\mathfrak{p}} \mathfrak{p}$ be an $S$-cycle of $K . \mathcal{I}_{S}^{\mathfrak{m}}$ is topologically generated by its subgroups $\left[K_{\mathfrak{p}}^{*}\right]_{\mathfrak{p}}, \mathfrak{p} \in S$, and $\left[U_{\mathfrak{p}}^{\left(m_{\mathfrak{p}}\right)}\right]_{\mathfrak{p}}, \mathfrak{p} \notin S$. But $K^{*} N_{L \mid K} \mathcal{I}_{L}$ is closed, hence the above facts may be summarized as

$$
\mathcal{I}_{S}^{\mathfrak{m}} \subseteq K^{*} N_{L \mid K} \mathcal{I}_{L} \quad \Leftrightarrow \quad \mathfrak{m} \geq \mathfrak{f}(L \mid K),
$$

which is what we had to show.

For a non-empty set $S$ of places of $K$ denote by $\operatorname{deg} S$ the greatest common divisor of the degrees of its elements.

1.3. Proposition. Let $S$ be a non-empty set of places and $\mathfrak{m}$ an $S$-cycle of $K$. Then $K_{S}^{\mathfrak{m}}$ is the largest abelian extension $L$ of $K$ such that $\mathfrak{f}(L \mid K) \leq \mathfrak{m}$ and every place in $S$ splits completely in L. Moreover, $\mathbb{F}_{q^{d}}$ with $d:=\operatorname{deg} S$ is the full constant field of $K_{S}^{\mathfrak{m}}$.

Proof. The maximality of $K_{S}^{\mathfrak{m}}$ with respect to the imposed conditions follows directly from the lemma (and its proof). As for the second assertion, consider the constant field extension $K_{d}=\mathbb{F}_{q^{d}} K$ of degree $d$ over $K$. It is 
unramified and such that all the places in $S$ are completely decomposed. Hence, by the maximality just proven, $\mathbb{F}_{q^{d}} \subseteq K_{d} \subseteq K_{S}^{\mathfrak{o}} \subseteq K_{S}^{\mathfrak{m}}$. On the other hand, in order that $\mathbb{F}_{q^{d^{\prime}}}$ for some $d^{\prime} \in \mathbb{N}$ is contained in $K_{S}^{\mathfrak{m}}, d^{\prime}$ must divide the degree of every place splitting completely in $K_{S}^{\mathfrak{m}}$.

For a finite abelian extension $L \mid K$, a place $\mathfrak{p}$ of $K$ and $n \geq-1$ we denote by $L^{n}(\mathfrak{p})$ the subfield of $L$ fixed by $G^{n}(L, \mathfrak{p})$ and call it the $n$th upper ramification field of $\mathfrak{p}$ in $L$. Given a set $S$ of places of $K$, any effective divisor $\mathfrak{m}$ of $K$ can always be made an $S$-cycle, denoted by $\mathfrak{m} \backslash S$, by removing all the places in $S$ from its support. With this notation we can state the remarkable fact that the ramification fields of the $S$-ray class field $\bmod \mathfrak{m}$ are the $S$-ray class fields mod $\mathfrak{m}^{\prime}$ for certain $\mathfrak{m}^{\prime} \leq \mathfrak{m}$.

1.4. TheOrem. Let $S$ be a non-empty set of places of $K, \mathfrak{m}=\sum_{\mathfrak{p}} m_{\mathfrak{p}} \mathfrak{p}$ an $S$-cycle and $L$ an intermediate field of $K_{S}^{\mathfrak{m}} \mid K$.

(a) For any place $\mathfrak{p}$ of $K$ and any $n \in \mathbb{N}_{0}$ we have

$$
L^{n}(\mathfrak{p})=L \cap K_{S}^{\mathfrak{m} \backslash\{\mathfrak{p}\}+n \mathfrak{p}} \quad \text { and } \quad L^{-1}(\mathfrak{p})=L \cap K_{S \cup\{\mathfrak{p}\}}^{\mathfrak{m} \backslash\{\mathfrak{p}\}}=L \cap K_{\{\mathfrak{p}\}}^{\mathfrak{m} \backslash\{\mathfrak{p}\}} .
$$

(b) The discriminant $\mathfrak{d}(L \mid K)$ of $L \mid K$ satisfies

$$
\mathfrak{d}(L \mid K)=[L: K] \mathfrak{m}-\sum_{\mathfrak{p}}\left(\sum_{n=0}^{m_{\mathfrak{p}}-1}\left[L^{n}(\mathfrak{p}): K\right]\right) \mathfrak{p} .
$$

P r o o f. (a) Consider an arbitrary intermediate field $L^{\prime}$ of $L \mid K$. According to $\left[16\right.$, p. 190] the restriction map $G(L \mid K) \rightarrow G\left(L^{\prime} \mid K\right)$ sends $G^{n}(L, \mathfrak{p})$ onto $G^{n}\left(L^{\prime}, \mathfrak{p}\right)$. Together with the previous proposition we obtain the following equivalence of conditions on $L^{\prime}$ :

$$
\begin{gathered}
L^{\prime} \subseteq L^{n}(\mathfrak{p}) \quad \Leftrightarrow \quad G\left(L \mid L^{\prime}\right) \supseteq G^{n}(L, \mathfrak{p}) \quad \Leftrightarrow \quad G^{n}\left(L^{\prime}, \mathfrak{p}\right)=1 \quad \Leftrightarrow \\
n \geq f\left(L^{\prime}, \mathfrak{p}\right) \quad \Leftrightarrow \quad \mathfrak{m} \backslash\{\mathfrak{p}\}+n \mathfrak{p} \geq \mathfrak{f}\left(L^{\prime} \mid K\right) \quad \Leftrightarrow \quad L^{\prime} \subseteq L \cap K_{S}^{\mathfrak{m} \backslash\{\mathfrak{p}\}+n \mathfrak{p} .}
\end{gathered}
$$

The proof for the decomposition field $L^{-1}(\mathfrak{p})$ is similar.

(b) For a place $\mathfrak{p}$ of $K$ with extension $\mathfrak{q}$ to $L$ denote by $d(\mathfrak{q} \mid \mathfrak{p})$ the different exponent and by $f(\mathfrak{q} \mid \mathfrak{p})$ the inertia degree of $\mathfrak{q} \mid \mathfrak{p}$, which should not be confused with the conductor exponent $f(L, \mathfrak{p})$. Using the Hasse-Arf Theorem one can rewrite Hilbert's (lower index) different formula in upper index form (for details see the appendix):

$$
d(\mathfrak{q} \mid \mathfrak{p})=\sum_{n=0}^{m_{\mathfrak{p}}-1}\left(\left[L: L^{0}(\mathfrak{p})\right]-\left[L^{n}(\mathfrak{p}): L^{0}(\mathfrak{p})\right]\right) .
$$

The discriminant is the norm of the different. Hence $\mathfrak{d}(L \mid K)=\sum_{\mathfrak{p}} d(L, \mathfrak{p}) \mathfrak{p}$ with 


$$
d(L, \mathfrak{p})=\sum_{\mathfrak{q} \mid \mathfrak{p}} f(\mathfrak{q} \mid \mathfrak{p}) d(\mathfrak{q} \mid \mathfrak{p})=[L: K] m_{\mathfrak{p}}-\sum_{n=0}^{m_{\mathfrak{p}}-1}\left[L^{n}(\mathfrak{p}): K\right],
$$

again using that $L^{0}(\mathfrak{p})$ is the inertia field of $\mathfrak{p}$ in $L$.

Thus computation of the discriminant (and thereby of the genus) of ray class field extensions amounts to determining their degrees. This has already been seen by Cohen et al. [3] in the number field case. As an illustration we give the following

1.5. Example (Ray class fields à la Hayes). Assume that $S$ consists of exactly one place of degree $d \in \mathbb{N}$. Let $\mathfrak{m}=\sum_{\mathfrak{p}} m_{\mathfrak{p}} \mathfrak{p}>\mathfrak{o}$ be an $S$-cycle, $h$ the (divisor) class number and $g$ the genus of $K$. Putting $\phi(\mathfrak{m}):=\left(\mathcal{I}_{S}^{\mathfrak{o}}: \mathcal{I}_{S}^{\mathfrak{m}}\right)=$ $\prod_{m_{\mathfrak{p}}>0}\left(q^{\operatorname{deg} \mathfrak{p}}-1\right) q^{\left(m_{\mathfrak{p}}-1\right) \operatorname{deg} \mathfrak{p}}$ we have:

(a) $\left[K_{S}^{\mathfrak{o}}: K\right]=h \cdot d$ and $\left[K_{S}^{\mathfrak{m}}: K\right]=h \cdot d \cdot \phi(\mathfrak{m}) /(q-1)$.

(b) The genus of $K_{S}^{\mathfrak{o}}$ is $g\left(K_{S}^{\mathfrak{o}}\right)=1+h(g-1)$. That of $K_{S}^{\mathfrak{m}}$ is

$$
g\left(K_{S}^{\mathfrak{m}}\right)=1+h \cdot[\phi(\mathfrak{m})(2 g-2+\operatorname{deg} \mathfrak{m})-s] /(2 q-2)
$$

with $s=(\phi(\mathfrak{m}) / \phi(\mathfrak{p})+q-2) \operatorname{deg} \mathfrak{p}$ if $\mathfrak{m}$ is the multiple of a single place $\mathfrak{p}$ and $s=\sum_{m_{\mathfrak{p}}>0} \phi(\mathfrak{m}) \operatorname{deg} \mathfrak{p} / \phi(\mathfrak{p})$ otherwise.

Proof. In order to verify the formula for $\phi(\mathfrak{m})$ look at the proof of Proposition 1.1 and recall that $\left(U_{\mathfrak{p}}: U_{\mathfrak{p}}^{(n)}\right)=\left(q^{\operatorname{deg} \mathfrak{p}}-1\right) q^{(n-1) \operatorname{deg} \mathfrak{p}}$ for each place $\mathfrak{p}$ and $n \in \mathbb{N}$.

(a) $|S|=1$ implies $h_{S}=h d$ and $\mathcal{O}_{S}^{*}=\mathbb{F}_{q}^{*}$, which is mapped injectively into $\mathcal{I}_{S}^{\mathfrak{o}} / \mathcal{I}_{S}^{\mathfrak{m}}$ because $\mathfrak{m}>\mathfrak{o}$. Hence the degree formulas follow from the exact sequence in Proposition 1.1.

(b) If $\operatorname{deg} \mathfrak{m}=1$ then $\mathfrak{f}\left(K_{S}^{\mathfrak{m}} \mid K\right)=\mathfrak{d}\left(K_{S}^{\mathfrak{m}} \mid K\right)=\mathfrak{o}$ by (a). Let $\operatorname{deg} \mathfrak{m}>1$ and $m_{\mathfrak{p}}>0$. Then

$$
\begin{aligned}
\sum_{n=1}^{m_{\mathfrak{p}}-1}\left[K_{S}^{\mathfrak{m} \backslash\{\mathfrak{p}\}+n \mathfrak{p}}: K\right] & =\frac{h d}{q-1} \sum_{n=1}^{m_{\mathfrak{p}}-1} \phi(\mathfrak{m}) q^{\left(n-m_{\mathfrak{p}}\right) \operatorname{deg} \mathfrak{p}} \\
& =\frac{h d}{q-1} \cdot \frac{\phi(\mathfrak{m})}{\phi(\mathfrak{p})}\left(1-q^{\left(1-m_{\mathfrak{p}}\right) \operatorname{deg} \mathfrak{p}}\right),
\end{aligned}
$$

and $\left[K_{S}^{\mathfrak{m} \backslash\{\mathfrak{p}\}}: K\right]$ is $h d$ or $\frac{h d}{q-1} \cdot \frac{\phi(\mathfrak{m})}{\phi\left(m_{\mathfrak{p}} \mathfrak{p}\right)}$ depending on whether or not $\mathfrak{m}$ is a multiple of $\mathfrak{p}$. If we apply Theorem 1.4, all this results in $\mathfrak{d}\left(K_{S}^{\mathfrak{m}} \mid K\right)=$ $h d(\phi(\mathfrak{m}) \mathfrak{m}-\mathfrak{s}) /(q-1)$ with $\mathfrak{s}=(\phi(\mathfrak{m}) / \phi(\mathfrak{p})+q-2) \mathfrak{p}$ if $\mathfrak{m}$ is supported by merely one place $\mathfrak{p}$ and $\mathfrak{s}=\sum_{m_{\mathfrak{p}}>0}(\phi(\mathfrak{m}) / \phi(\mathfrak{p})) \mathfrak{p}$ otherwise. The genus is now easily computed from the degree and the discriminant by means of the Hurwitz genus formula. (See [37, p. 88] and take into consideration that $K_{S}^{\mathfrak{m}}$ has constant field $\mathbb{F}_{q^{d}}$.) 
As soon as $S$ consists of more than one place, the determination of the degrees is much more difficult. We shall dedicate Section 3 to working out this problem in a special case.

2. Upper bounds. As before let $K$ be a global function field with full constant field $\mathbb{F}_{q}$. By the Hasse-Weil Theorem (see [37, pp. 169ff]) as a first estimate for its number $N(K)$ of rational places we obtain

$$
N(K) \leq q+1+2 g(K) \sqrt{q},
$$

where $g(K)$ denotes the genus of $K$. This is called the Hasse-Weil bound. In case equality holds, $K$ is called maximal. Obviously $K$ can only be maximal if $g(K)=0$ or if $q$ is a square. A consideration involving the constant field extension of degree 2 (see [37, p. 182]) and a recent work by Fuhrmann and Torres [5] shows that in addition one must have $g(K)=(q-\sqrt{q}) / 2$ or $g(K) \leq$ $(\sqrt{q}-1)^{2} / 4$. In the non-square case Serre [34] was able to improve (1) to

$$
N(K) \leq q+1+g(K)\lfloor 2 \sqrt{q}\rfloor
$$

where $\lfloor a\rfloor$ denotes the integer part of $a \in \mathbb{R}$.

The maximum number of rational places a global function field of genus $g$ with full constant field $\mathbb{F}_{q}$ can have is usually denoted by $N_{q}(g)$. We call $K$ optimal if $N(K)=N_{q}(g(K))$. Serre [36] succeeded in determining $N_{q}(g)$ for $g=1$ and 2 in general and in some cases also for $g=3$ and 4 . An overview of what is known about $N_{q}(g)$ for $g \leq 50$ and $q$ a not too large power of 2 or 3 is found in [9], [17], [27] or [28].

For large genera one can improve (1) by means of the so-called explicit Weil formulas (see [37, p. 183]): Any finite sequence $\left(c_{n}\right)$ of reals $c_{n} \geq 0$ satisfying

$$
1+\sum_{n \geq 1} 2 c_{n} \cos n \varphi \geq 0 \quad \forall \varphi \in \mathbb{R}
$$

provides an estimate

$$
g(K) \geq(N(K)-1) \sum_{n \geq 1} c_{n} q^{-n / 2}-\sum_{n \geq 1} c_{n} q^{n / 2} .
$$

We shall give a brief outline of the maximization of the right hand side due to Oesterlé as found in [33] and, based on it, derive an estimate for $S$-class numbers that spares us from going into the explicit formulas again and again.

Given a real number $N \geq q+1$, let $m$ be the integer $\geq 2$ such that $q^{m / 2} \leq N-1<q^{(m+1) / 2}$ and

$$
u:=\left[\left(q^{(m+1) / 2}-q^{(m-1) / 2}\right) /\left(N-1-q^{(m-1) / 2}\right)-1\right] / \sqrt{q} \in(0,1] .
$$


Because the continuous maps

$$
F_{m}:\left[\frac{\pi}{m+1}, \frac{\pi}{m}\right] \rightarrow[0,1], \quad \varphi \mapsto-\left(\cos \frac{m+1}{2} \varphi\right) /\left(\cos \frac{m-1}{2} \varphi\right),
$$

are onto and strictly increasing, $\vartheta_{q}(N):=\cos \left(F_{m}^{-1}(u)\right)$ defines a continuous bijection $\vartheta_{q}:[q+1, \infty) \rightarrow[0,1)$, which is also increasing.

\subsection{Theorem (Oesterlé). Define}

$$
g_{q}:[q+1, \infty) \rightarrow[0, \infty), \quad N \mapsto 1+\frac{\left(\sqrt{q} \vartheta_{q}(N)-1\right) N}{q-2 \sqrt{q} \vartheta_{q}(N)+1},
$$

and assume that $N(K) \geq q+1$. Then $g(K) \geq g_{q}(N(K))$, and $g_{q}(N(K))$ is the maximum of the right hand side of (2) for $q \geq 3$ (but not always for $q=2$ ).

After checking that $g_{q}$ is strictly increasing we find that the value at $g \in \mathbb{N}_{0}$ of its inverse $\bar{N}_{q}:=g_{q}^{-1}$ provides an upper bound for the number $N_{q}(g)$, which for $g \leq(q-\sqrt{q}) / 2$ coincides with the Hasse-Weil bound (1) and improves it for larger $g . \bar{N}_{q}(g)$ will be called the Oesterlé bound.

Now let $S$ be a non-empty set of places of $K$. Applying Oesterlé's Theorem to the Hilbert class field of $\mathcal{O}_{S}$ yields an upper bound for the $S$-class number $h_{S}$.

2.2. Lemma. Assume that $K$ has genus $g \geq 1$ and that $S$ contains at least $N>(\sqrt{q}-1)(g-1)$ rational places. Then

$$
h_{S} \leq \hbar_{q}(g, N):=\vartheta_{q}^{-1}(t) / N \quad \text { with } \quad t:=\left(1+\frac{(q-1)(g-1)}{N+2 g-2}\right) / \sqrt{q} .
$$

Proof. The restrictions on $g$ and $N$ ensure that $1 / \sqrt{q} \leq t<1$, whereby $\hbar_{q}$ is well defined. Let $g^{\prime}:=g\left(K_{S}^{\mathfrak{o}}\right)$ and $N^{\prime}:=N\left(K_{S}^{\mathfrak{o}}\right)$ be the genus resp. the number of rational places of the Hilbert class field of $\mathcal{O}_{S}$, which satisfy $h_{S}(g-1)=g^{\prime}-1$ and $h_{S} N \leq N^{\prime}$. For $N^{\prime} \leq q+1$ the claimed estimate is obvious. Assume $N^{\prime} \geq q+1$. Then by Oesterlé's Theorem,

$$
\frac{g-1}{N} \geq \frac{g^{\prime}-1}{N^{\prime}} \geq \frac{g_{q}\left(N^{\prime}\right)-1}{N^{\prime}}=\frac{\sqrt{q} \vartheta_{q}\left(N^{\prime}\right)-1}{q-2 \sqrt{q} \vartheta_{q}\left(N^{\prime}\right)+1} .
$$

Isolating $\vartheta_{q}\left(N^{\prime}\right)$ in this inequality results in $\vartheta_{q}\left(N^{\prime}\right) \leq t$, from which by the isotony of $\vartheta_{q}$ we get the assertion.

Note that $\vartheta_{q}^{-1}$ can be computed very efficiently using the cosine formula for multiple angles. In fact, on each interval $\left[\cos \frac{\pi}{m}, \cos \frac{\pi}{m+1}\right]$ with $m$ being an integer $\geq 2$ it is identical with a $\mathbb{Q}(\sqrt{q})$-rational function, namely

$$
\vartheta_{q}^{-1}(t)=1+\left(q^{(m+1) / 2}+q^{m / 2} u\right) /(1+\sqrt{q} u) \text { with } u=f_{m+1}(t) / f_{m-1}(t),
$$


where

$$
f_{n}(t):=\sum_{i=0}^{\lfloor n / 2\rfloor} \frac{n}{n-i}\left(\begin{array}{c}
n-i \\
i
\end{array}\right)(-2 t-2)^{\lfloor n / 2\rfloor-i} \quad \text { for } n \in \mathbb{N} .
$$

This also shows $\hbar_{q}(g, N) \in \mathbb{Q}(\sqrt{q})$.

An essential step in determining the degrees of the $S$-ray class fields is the computation of $h_{S}$. This can often be done using Lemma 2.2 and the relation of $h_{S}$ with the $S$-regulator $\operatorname{reg}_{S}$, defined as the index of the group $\left(\mathcal{O}_{S}^{*}\right)$ of principal divisors of $S$-units in the group $\mathcal{D}_{S}^{0}$ of divisors of degree 0 with support in $S$ (see [31] for details).

2.3. Example. Consider the function field $K:=\mathbb{F}_{2}(x, y)$ with $y^{2}+y=$ $x^{3}(x+1)^{2}$ of genus $g(K)=2$. It has 5 places of degree 1 and no place of degree 2 , whence its divisor class number is $h(K)=13$. Let $\mathfrak{p}_{\infty}$ be the pole of $x$ (and $y)$ and put $\mathfrak{p}_{0}:=(0,0), \mathfrak{p}_{1}:=(0,1), \mathfrak{p}_{2}:=(1,0)$ and $\mathfrak{p}_{3}:=(1,1)$, where $(\alpha, \beta)$ denotes the common zero of $x-\alpha$ and $y-\beta$. First look at the set $S:=\left\{\mathfrak{p}_{\infty}, \mathfrak{p}_{0}, \ldots, \mathfrak{p}_{3}\right\}$ of all rational places of $K$. Since $\hbar_{2}(2,5)=$ $(2503+960 \sqrt{2}) / 2911<2$ and $h_{S} \cdot \operatorname{reg}_{S}=h(K)$ we see that $h_{S}=1$ and $\operatorname{reg}_{S}=13$. Let $O$ be the subgroup of $\mathcal{O}_{S}^{*}$ generated by $x, x+1, y$ and $y+x^{2}$. Without regard to sign, the index of $(O)$ in $\mathcal{D}_{S}^{0}$ equals any $4 \times 4$-minor of the matrix

$$
D:=\left(v_{\mathfrak{p}_{j}}(z)\right)_{\substack{z=x, x+1, y, y+x^{2} \\
j=\infty, 1,2,3,0}}=\left(\begin{array}{ccccc}
-2 & 1 & 0 & 0 & 1 \\
-2 & 0 & 1 & 1 & 0 \\
-5 & 0 & 2 & 0 & 3 \\
-5 & 0 & 0 & 3 & 2
\end{array}\right),
$$

which turns out to be \pm 13 . Hence $\mathcal{O}_{S}^{*}=O$. Now we can compute $h_{S^{\prime}}$ and a basis of $\mathcal{O}_{S^{\prime}}^{*}$ for any $S^{\prime} \subseteq S$, e.g. for $S_{r}:=\left\{\mathfrak{p}_{\infty}, \mathfrak{p}_{1}, \ldots, \mathfrak{p}_{r}\right\}$ the first $r \in\{1,2,3\}$ rows of the Hermite normal form

$$
\left(\begin{array}{rrrr}
13 & 6 & -3 & -2 \\
5 & 3 & -1 & -1 \\
-5 & -2 & 1 & 1 \\
1 & 0 & 0 & 0
\end{array}\right) \cdot D=\left(\begin{array}{rrrrr}
-13 & 13 & 0 & 0 & 0 \\
-6 & 5 & 1 & 0 & 0 \\
4 & -5 & 0 & 1 & 0 \\
-2 & 1 & 0 & 0 & 1
\end{array}\right)
$$

considered as elements of $\mathcal{D}_{S}^{0}$ constitute a basis of $\left(\mathcal{O}_{S_{r}}^{*}\right)$, so we see that $h_{S_{r}}=1$. This example is continued in part (B) of Section 3 .

3. Many rational places. When searching for curves with many rational points, it seems to be fruitful to investigate ray class fields $K_{S}^{\mathfrak{m}}$, where $K$ already has many rational places compared to its genus, several or all of which are contained in $S$.

First we want to fix some notation. Let $K \mid \mathbb{F}_{q}$ be as before, and let $g(K)$ be the genus and $p$ the characteristic of $K$. Then $q=p^{e}$ for some $e \in \mathbb{N}$. By 
$v_{p}$ we denote the (normalized) $p$-adic valuation on $\mathbb{Q}$ or its $p$-adic completion $\mathbb{Q}_{p}$ with values in $\mathbb{Z} \cup\{\infty\}$. Throughout this whole section, $n$ always denotes a positive (or, where this makes sense, a non-negative) integer. For $n \geq 1$ we put $n^{*}:=n / p^{v_{p}(n)}$.

To simplify the situation somewhat we restrict ourselves to the case of $\mathfrak{m}$ being the multiple of a single rational place $\mathfrak{p}$ of $K\left({ }^{1}\right)$. Let $S$ be a non-empty set of places of $K$ not containing p. Put $U^{(n)}:=U_{\mathfrak{p}}^{(n)}$ and $U_{S}:=\mathcal{O}_{S}^{*} \cap U^{(1)}$. By Dirichlet's Unit Theorem $U_{S} \simeq \mathcal{O}_{S}^{*} / \mathbb{F}_{q}^{*}$ is a free abelian group of rank $|S|-1$. Since $U^{(0)}=\mathbb{F}_{q}^{*} U^{(1)}$ and $\mathbb{F}_{q}^{*}$ is contained in $\mathcal{O}_{S}^{*}$, the exact sequence of Proposition 1.1 yields $K_{S}^{\mathfrak{o}}=K_{S}^{\mathfrak{p}}$, and using the isomorphisms discussed in Section 1, it can be rewritten as

$$
1 \rightarrow U_{S} \cap U^{(n)} \rightarrow U_{S} \rightarrow U^{(1)} / U^{(n)} \rightarrow G\left(K_{S}^{n \mathfrak{p}} \mid K\right) \rightarrow \mathcal{C}\left(\mathcal{O}_{S}\right) \rightarrow 1,
$$

whence $G\left(K_{S}^{n \mathfrak{p}} \mid K_{S}^{\mathfrak{o}}\right) \simeq U^{(1)} / U_{S} U^{(n)}$. Let $\lambda_{S}^{(n)}:=\log _{p}\left[K_{S}^{n \mathfrak{p}}: K_{S}^{\mathfrak{o}}\right]$, where $\log _{p}$ means logarithm to the base $p$. Then clearly $\lambda_{S}^{(0)}=\lambda_{S}^{(1)}=0$ and $\lambda_{S}^{(n)}=\log _{p}\left(U^{(1)}: U_{S} U^{(n)}\right) \in \mathbb{N}_{0}$ for $n \geq 1$. Wanting to have many examples, for each $l \in\left\{\lambda_{S}^{(n-1)}, \ldots, \lambda_{S}^{(n)}\right\}$ by Galois theory we choose an intermediate field $L_{l, S}$ of $K_{S}^{n \mathfrak{p}} \mid K_{S}^{(n-1) \mathfrak{p}}$ of degree $p^{l}$ over $K_{S}^{\mathfrak{o}}$. Assume $\operatorname{deg} S=1$, then by Proposition 1.3 each $L_{l, S}$ has again full constant field $\mathbb{F}_{q}$ and Theorem 1.4 together with the Hurwitz genus formula yields

$$
g\left(L_{l, S}\right)=1+\frac{h_{S}}{2}\left(p^{l}(2 g(K)-2+n)-\sum_{\nu=0}^{n-1} p^{\lambda_{S}^{(\nu)}}\right) .
$$

Since $h_{S} / h_{S \cup\{\mathfrak{p}\}}$ is the inertia degree of $\mathfrak{p}$ in $L_{l, S}$, the number $N\left(L_{l, S}\right)$ of rational places of $L_{l, S}$ satisfies

$$
N\left(L_{l, S}\right) \geq h_{S} \cdot p^{l} \cdot|\{\mathfrak{q} \in S \mid \operatorname{deg} \mathfrak{q}=1\}|+ \begin{cases}h_{S} & \text { if } h_{S}=h_{S \cup\{\mathfrak{p}\}}, \\ 0 & \text { if } h_{S}>h_{S \cup\{\mathfrak{p}\}},\end{cases}
$$

where equality holds if and only if $L_{l, S} \nsubseteq K_{S \cup\{\mathfrak{q}\}}^{n \mathfrak{p}}$ (which is guaranteed for $\left.l>\lambda_{S \cup\{\mathfrak{q}\}}^{(n)}\right)$ for each rational place $\mathfrak{q}$ not contained in $S \cup\{\mathfrak{p}\}$.

We shall give two methods to determine the degrees of the $K_{S}^{n \mathfrak{p}}$ over $K$. Method (A) is specialized to the rational function field and can be carried out very easily in practice. Method (B) means much more effort but applies to arbitrary $K$.

(A) Rational function field. First we investigate the case $K=\mathbb{F}_{q}(x)$, where $x$ is an indeterminate over $\mathbb{F}_{q}$. Also let $S$ consist of rational places only (implying $h_{S}=h(K)=1$, so we have $K_{S}^{\mathfrak{o}}=K$ ). For $\alpha \in \mathbb{F}_{q}$ denote the zero of $x-\alpha$ by $\mathfrak{p}_{\alpha}$. After an appropriate transformation of the variable

$\left({ }^{1}\right)$ The more general case of $\mathfrak{p}$ having arbitrary degree is treated in [1]. 
$x$ we can assume that $\mathfrak{p}$ is the pole of $x$ and that $\mathfrak{p}_{0}$ is contained in $S$. Then $\pi:=1 / x$ is a uniformizer at $\mathfrak{p}$, and $U_{S}$ is freely generated by the functions $1-\alpha \pi$ with $\alpha \in A_{S}:=\left\{\alpha \in \mathbb{F}_{q}^{*} \mid \mathfrak{p}_{\alpha} \in S\right\}$.

By what has been said above, our task is to determine the logarithmic degrees $\lambda_{S}^{(n)}=\log _{p}\left[K_{S}^{n \mathfrak{p}}: K\right]$. In many cases they obey a very simple rule (Theorem 3.3 below), which can be developed by the following idea involving Newton's Formulas.

Choose a generator $\omega$ of $\mathbb{F}_{q}^{*}$, put $I:=\{1, \ldots, q-1\}$ and $I_{S}:=\{j \in$ $\left.I \mid \omega^{j} \in A_{S}\right\}$, let ${ }^{-}$denote the canonical projection

$$
\mathbb{F}_{p}[x] \rightarrow R:=\mathbb{F}_{p}[x] /\left(x^{q-1}-1\right)
$$

and define the morphism

$$
\varrho: U_{S} \rightarrow R, \quad \prod_{\alpha \in A_{S}}(1-\alpha \pi)^{c_{\alpha}} \mapsto \sum_{j \in I_{S}} c_{\omega^{j}} \bar{x}^{j},
$$

of groups with kernel $U_{S}^{p}$ and image $R_{S}:=\bigoplus_{j \in I_{S}} \mathbb{F}_{p} \bar{x}^{j}$.

Denote by $f_{\alpha} \in \mathbb{F}_{p}[x]$ the minimal polynomial of $\alpha \in \mathbb{F}_{q}$ over $\mathbb{F}_{p}$. The ring $R$ is frequently used in coding theory. It is principal (as homomorphic image of $\mathbb{F}_{p}[x]$ ) and its ideals (which are in fact cyclic codes) correspond bijectively to the monic divisors of $x^{q-1}-1$. Hence the kernel of the $\mathbb{F}_{p^{-}}$ algebra morphism

$$
\Phi^{(n)}: R \rightarrow \mathbb{F}_{q}^{n-1}, \quad \bar{f} \mapsto\left(f\left(\omega^{i}\right)\right)_{1 \leq i<n},
$$

is just the ideal $R \bar{f}^{(n)}$, where $f^{(n)}$ is the least common multiple of all $f_{\omega^{i}}$ with $1 \leq i<n$.

Denote the degree of $f^{(n+1)} / f^{(n)}$ by $e^{(n)}$ and call $n$ (an) initial (of $q$ ) if $e^{(n)}>0$. Let $G\left(\mathbb{F}_{q} \mid \mathbb{F}_{p}\right)$ act on $I$ via the bijection $I \leftrightarrow \mathbb{F}_{q}^{*}, i \mapsto \omega^{i}$, and consider the orbits under this action. Obviously $n$ is initial iff it is in $I$ and is the least element in its orbit, in which case $e^{(n)}=\operatorname{deg} f_{\omega^{n}}=\left[\mathbb{F}_{p}\left(\omega^{n}\right): \mathbb{F}_{p}\right]$ is the length of this orbit and divides $e$. For example if $q=16, I$ decomposes into the orbits $\{1,2,4,8\},\{3,6,9,12\},\{5,10\},\{7,11,13,14\}$ and $\{15\}$, which means that the initials of $q=16$ are $1,3,5,7$ and 15 , where $e^{(1)}=e^{(3)}=$ $e^{(7)}=4, e^{(5)}=2$ and $e^{(15)}=1$.

Let $\varphi$ be the Frobenius on $K$, which takes everything to its $p$ th power. If we write the elements of $I$ in $p$-adic representation with $e$ digits, the action of $\varphi^{l}$ is just a rotation of these digits by $l$ places. If we use this to determine whether or not $n$ is initial, the proof of the following lemma is easy and can therefore be omitted.

3.1. LEMMA. (a) $f^{(n)}=x^{q-1}-1$ iff $n \geq q$.

(b) $f^{(n)}=1+\ldots+x^{q-2}$ iff $q-q / p \leq n<q$. 
(c) If $p$ divides $n$ then $e^{(n)}=0$.

(d) If $e$ is even and $n \in\{1, \ldots, 2 \sqrt{q}\} \backslash(\mathbb{Z} p \cup\{\sqrt{q}+1\})$, then $e^{(n)}=e$, $e^{(\sqrt{q}+1)}=e / 2$ and $e^{(2 \sqrt{q}+1)}=0$.

(e) If $e$ is odd, then $e^{(n)}=e$ for $n \in\{1, \ldots, \sqrt{p q}\} \backslash \mathbb{Z} p$ and $e^{(n)}=0$ for $n \in\{\sqrt{p q}, \ldots, \sqrt{p q}+p\}$.

Put $n^{\prime}:=\lceil n / p\rceil$, where $\lceil u\rceil$ denotes the least integer greater than or equal to $u$. Then the Frobenius map induces an injection $\varphi^{(n)}: U^{(1)} / U^{\left(n^{\prime}\right)} \hookrightarrow$ $U^{(1)} / U^{(n)}$. To be able to continue we must impose certain restrictions on $n^{\prime}$ and $n$ in terms of the two numbers

$$
\begin{aligned}
& n_{S}^{\prime}:=\max \left\{n \in \mathbb{N} \mid \lambda_{S}^{(n)}=0\right\}=\max \left\{n \in \mathbb{N} \mid U^{(1)}=U_{S} U^{(n)}\right\}, \\
& n_{S}:=\min \left\{q-q / p, n \in \mathbb{N} \mid R_{S} \cap R \bar{f}^{(n)}=0\right\}-1 .
\end{aligned}
$$

The main work will be done in the following

3.2. Lemma. (a) Let $y \in U_{S}$. Then $y U^{(n)}$ lies in the image of $\varphi^{(n)}$ if and only if $\varrho(y) \in R \bar{f}^{(n)}$.

(b) Assume that $n^{\prime} \leq n_{S}^{\prime}$ or $n>n_{S}$. Then

$$
\frac{\left(U_{S} U^{(n)}: U^{(n)}\right)}{\left(U_{S} U^{\left(n^{\prime}\right)}: U^{\left(n^{\prime}\right)}\right)}=\left(R_{S}: R_{S} \cap R \bar{f}^{(n)}\right) .
$$

Proof. (a) Write $y=\prod_{\alpha \in A_{S}}(1-\alpha \pi)^{c_{\alpha}}$ with integers $c_{\alpha}$, let

$$
y=\sum_{j=0}^{\infty} \sigma_{j} \pi^{j}, \quad \sigma_{j} \in \mathbb{F}_{q},
$$

be the expansion of $y$ at $\mathfrak{p}$ and put $s_{i}:=\sum_{\alpha \in A_{S}} c_{\alpha} \alpha^{i}$. Since multiplication of $y$ by $(1-\alpha \pi)^{p^{l}}$ neither changes the $s_{i}$ nor $\sigma_{0}, \ldots, \sigma_{n}$ provided $n<p^{l}$ and $l \in \mathbb{N}$, we can assume that none of the $c_{\alpha}$ is negative and obtain

$$
n \sigma_{n}+\sum_{i=1}^{n} s_{i} \sigma_{n-i}=0
$$

from Newton's Formulas. By induction we deduce the equivalence

$$
\forall j \in\{1, \ldots, n-1\} \backslash \mathbb{Z} p: \sigma_{j}=0 \quad \Leftrightarrow \quad s_{1}=\ldots=s_{n-1}=0 .
$$

Since $\Phi^{(n)}(\varrho(y))=\left(s_{i}\right)_{1 \leq i<n}$, this can be reformulated to yield the assertion.

(b) All the above defined morphisms can be arranged in a commutative 
diagram

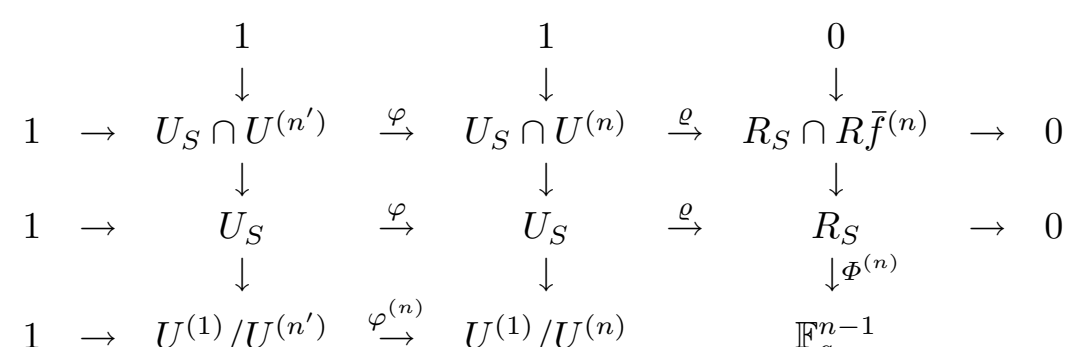

(use (a) to see that $\left.\varrho\left(U_{S} \cap U^{(n)}\right) \subseteq R \bar{f}^{(n)}\right)$, the exactness of which is easily verified except at one critical point, namely the horizontal passage through the upper right corner, i.e. it remains to show that $\varrho\left(U_{S} \cap U^{(n)}\right)$ really is all of $R_{S} \cap R \bar{f}^{(n)}$. To this end let $y \in U_{S}$ with $\varrho(y) \in R \bar{f}^{(n)}$.

First assume that $n^{\prime} \leq n_{S}^{\prime}$. Then by (a) there exists $y^{\prime} \in U_{S}$ with $y U^{(n)}=\varphi^{(n)}\left(y^{\prime} U^{\left(n^{\prime}\right)}\right)=y^{\prime p} U^{(n)}$, i.e. $y / y^{\prime p} \in U^{(n)}$. But $\varrho\left(y / y^{\prime p}\right)=\varrho(y)$ from the exactness of the middle row.

By Lemma 3.1(a),(b), $R_{S} \cap R \bar{f}^{(n)}$ is trivial if $n \geq q$ and also if $n>n_{S}$ and $|S|<q$. Finally, if $q-q / p \leq n<q$ and $|S|=q$, from Lemma 3.1(b) we know that $\varrho(y)=\gamma\left(1+\ldots+x^{q-2}\right)$ for some $\gamma \in \mathbb{F}_{p}$, i.e. $y$ is a power of $1-\pi^{q-1}$ and therefore contained in $U^{(n)}$.

Now use the isomorphism

$$
U_{S} U^{(n)} / U^{(n)} \simeq \frac{U_{S} / \varphi\left(U_{S} \cap U^{\left(n^{\prime}\right)}\right)}{U_{S} \cap U^{(n)} / \varphi\left(U_{S} \cap U^{\left(n^{\prime}\right)}\right)}
$$

and the exactness just proven to obtain

$$
\begin{aligned}
\frac{\left(U_{S} U^{(n)}: U^{(n)}\right)}{\left(U_{S} U^{\left(n^{\prime}\right)}: U^{\left(n^{\prime}\right)}\right)} & =\frac{\left(U_{S}: \varphi\left(U_{S}\right)\right)\left(\varphi\left(U_{S}\right): \varphi\left(U_{S} \cap U^{\left(n^{\prime}\right)}\right)\right)}{\left(U_{S} \cap U^{(n)}: \varphi\left(U_{S} \cap U^{\left(n^{\prime}\right)}\right)\right)\left(U_{S}: U_{S} \cap U^{\left(n^{\prime}\right)}\right)} \\
& =\left(R_{S}: R_{S} \cap R \bar{f}^{(n)}\right) .
\end{aligned}
$$

We have arrived at the main theorem of Method (A). It gives a formula for the (logarithmic) degrees of $K_{S}^{n \mathfrak{p}} \mid K$ in terms of the non-p-part $n^{*}$ of $n$ defined above and the numbers

$$
e_{S}^{(n)}:=\operatorname{dim}_{\mathbb{F}_{p}}\left(R_{S} \cap R \bar{f}^{(n)} / R_{S} \cap R \bar{f}^{(n+1)}\right),
$$

which are $\leq e^{(n)}$ and whose computation is an easy exercise in linear algebra or even simpler. Lauter [15] has already proved this formula for all $n$ in case $|S|=q$ by a different method.

3.3. Theorem. (a) As long as $n<p n_{S}^{\prime}$ the $\lambda_{S}^{(n)}$ satisfy the recursion

$$
\lambda_{S}^{(n+1)}=\lambda_{S}^{(n)}+e-e_{S}^{\left(n^{*}\right)} .
$$

If $p n_{S}^{\prime} \geq n_{S}$ this formula holds for every $n \in \mathbb{N}$. 
(b) $n_{S}^{\prime}=\min \left\{n \in \mathbb{N} \mid e_{S}^{\left(n^{*}\right)}<e\right\}$ and

$$
n_{S}= \begin{cases}0 & \text { if }|S|=1, \\ \max \left\{n \in \mathbb{N} \mid e_{S}^{(n)}>0\right\} & \text { if } 1<|S|<q, \\ q-q / p-1 & \text { if }|S|=q .\end{cases}
$$

(c) If $e=1$, i.e. $q=p$, then

$$
e_{S}^{(n)}= \begin{cases}1 & \text { if } n<|S| \\ 0 & \text { otherwise }\end{cases}
$$

and the formula in (a) holds for every $n \in \mathbb{N}$.

Proof. (a) From the exact sequence (3) we obtain $\lambda_{S}^{(n)}=(n-1) e-$ $\log _{p}\left(U_{S} U^{(n)}: U^{(n)}\right)$. Hence it suffices to show that

$$
\log _{p} \frac{\left(U_{S} U^{(n+1)}: U^{(n+1)}\right)}{\left(U_{S} U^{(n)}: U^{(n)}\right)}=e_{S}^{\left(n^{*}\right)}
$$

under the given assumptions. But this is easily derived from the previous lemma by induction on $n$, distinguishing whether or not $p$ divides $n$.

(b) follows immediately from (a).

(c) Observe that

$$
\operatorname{dim}_{\mathbb{F}_{p}} \Phi^{(n)}\left(R_{S}\right)=\operatorname{rank}\left(\omega^{i j}\right)_{1 \leq i<n, j \in I_{S}}=\min \left\{n-1,\left|I_{S}\right|\right\},
$$

\begin{tabular}{|c|c|c|c|c|c|c|c|c|c|c|c|}
\hline \multicolumn{12}{|c|}{$q=2$} \\
\hline$l,|S|$ & 1,2 & 2,2 & 3,2 & 4,1 & 4,2 & 5,1 & 5,2 & 6,1 & 6,2 & 7,2 & 8,2 \\
\hline$g\left(L_{l, S}\right)$ & 1 & 5 & 15 & 17 & 39 & 49 & 103 & 129 & 247 & 567 & 1271 \\
\hline$N\left(L_{l, S}\right)$ & 5 & 9 & 17 & 17 & 33 & 33 & 65 & 65 & 129 & 257 & 513 \\
\hline \multicolumn{12}{|c|}{$q=3$} \\
\hline$l,|S|$ & 1,2 & 1,3 & 2,2 & 2,3 & 3,1 & 3,2 & 3,3 & 4,2 & 4,3 & 5,2 & 5,3 \\
\hline$g\left(L_{l, S}\right)$ & 1 & 3 & 10 & 15 & 21 & 46 & 69 & 181 & 258 & 667 & 987 \\
\hline$N\left(L_{l, S}\right)$ & 7 & 10 & 19 & 28 & 28 & 55 & 82 & 163 & 244 & 487 & 730 \\
\hline \multicolumn{12}{|c|}{$q=5$} \\
\hline$l,|S|$ & 1,2 & 1,3 & 1,4 & 1,5 & 2,2 & 2,3 & 2,4 & 2,5 & 3,2 & 3,3 & 3,4 \\
\hline$g\left(L_{l, S}\right)$ & 2 & 4 & 6 & 10 & 22 & 34 & 56 & 70 & 172 & 284 & 356 \\
\hline$N\left(L_{l, S}\right)$ & 11 & 16 & 21 & 26 & 51 & 76 & 101 & 126 & 251 & 376 & 501 \\
\hline \multicolumn{12}{|c|}{$q=7$} \\
\hline$l,|S|$ & 1,2 & 1,3 & 1,4 & 1,5 & 1,6 & 2,2 & 2,3 & 2,4 & 2,5 & 2,6 & 2,7 \\
\hline$g\left(L_{l, S}\right)$ & 3 & 6 & 9 & 12 & 15 & 45 & 69 & 93 & 117 & 162 & 189 \\
\hline$N\left(L_{l, S}\right)$ & 15 & 22 & 29 & 36 & 43 & 99 & 148 & 197 & 246 & 295 & 344 \\
\hline
\end{tabular}

showing the assertion on $e_{S}^{(n)}$, and use (b).

Table 1. Ray class fields of $\mathbb{F}_{q}(x)$ for $q=2,3,5$ and 7 
Without any further effort, Theorem 3.3(a) and (c) in combination with equations (4) and (5) leads to Table 1, giving $g\left(L_{l, S}\right)$ and $N\left(L_{l, S}\right)$ for some prime numbers $q=p$ and some values of $l$ and $|S|$. For $q=2$ examples from [38] are reproduced, and all entries with $|S|=1$ are known as cyclotomic function fields. For $q=3$ and $g\left(L_{l, S}\right) \geq 181$ we encounter first examples of new function fields with many rational places. The table for $q=5$ continues a series of examples worked out in [24] for genus up to 56. For $q=7$ all examples except the one with $g\left(L_{l, S}\right)=3$ are new. Writing down the tables for larger prime numbers is not worthwhile because the entries for $N\left(L_{l, S}\right)$ are getting comparatively small.

A direct computation shows that 1 and 3 are the initials of $q=4$ with $e_{S}^{(1)}=\min \{2,|S|-1\}$ and $e_{S}^{(3)}=\max \{0,|S|-3\}$, so Theorem 3.3 gives the values in Table 2. It yields some new estimates compared to [28], e.g. $97 \leq N_{4}(67) \leq 117$. A more complete table obtained by various other constructions is found e.g. in [20].

Table 2. Ray class fields of $\mathbb{F}_{4}(x)$, and intermediate fields

\begin{tabular}{lccccccccccc}
\hline \multicolumn{110}{c}{$q=4$} \\
\hline$l,|S|$ & 1,4 & 3,1 & 2,3 & 2,4 & 4,1 & 3,3 & 3,4 & 5,1 & 4,3 & 4,4 & 5,2 \\
$g\left(L_{l, S}\right)$ & 1 & 2 & 3 & 5 & 6 & 11 & 13 & 22 & 27 & 33 & 37 \\
$N\left(L_{l, S}\right)$ & 9 & 9 & 13 & 17 & 17 & 25 & 33 & 33 & 49 & 65 & 65 \\
\hline \hline$l,|S|$ & 5,3 & 5,4 & 6,2 & 6,3 & 6,4 & 7,2 & 7,3 & 7,4 & 8,3 & 8,4 \\
$g\left(L_{l, S}\right)$ & 67 & 81 & 101 & 147 & 177 & 229 & 339 & 433 & 723 & 945 \\
$N\left(L_{l, S}\right)$ & 97 & 129 & 129 & 193 & 257 & 257 & 385 & 513 & 769 & 1025 \\
\hline
\end{tabular}

While in the previous examples the $e_{S}^{(n)}$ only depended on $|S|$, they may vary for different $S$ in the same cardinality when regarding proper prime powers $q \neq 4$. Due to this fact we have to perform some linear algebra before computing the tables for $q=8,9,16,25,27,32,49,64$ and 81, which are found at the end of this section.

Let us now take a closer look at the situation when $|S|=q$. Put $r:=$ $\sqrt{q}$ or $\sqrt{p q}$ depending on whether $e$ is even or odd. From Theorem 3.3 and Lemma 3.1(c)-(e) we obtain two canonical fields, namely $K_{S}^{(r+2) \mathfrak{p}}$ and $K_{S}^{(2 r+2) \mathfrak{p}}$ of degree $r$ and $r q$ over $K$ if $e$ is even, and $p-1$ canonical fields $K_{S}^{(r+i+1) \mathfrak{p}}$ with $1 \leq i<p$ of degree $q^{i}$ over $K$ in case $e$ is odd. Their genera and those of all intermediate fields $L_{l, S}$ are easily computed from our formulas. 
3.4. Corollary. Let $|S|=q$. Then $N\left(L_{l, S}\right)=1+p^{l} q$ and

(a) $g\left(L_{l, S}\right)=\left\{\begin{array}{ll}\frac{r}{2}\left(p^{l}-1\right) & \text { for } 0 \leq l \leq e / 2, \\ \frac{r}{2}\left(2 p^{l}-r-1\right) & \text { for } e / 2 \leq l \leq 3 e / 2\end{array}\right\}$ if e is even,

(b) $g\left(L_{l, S}\right)=\frac{1}{2}\left(p^{l}(r+i-1)-r-\frac{q^{i}-q}{q-1}\right)$ for $(i-1) e \leq l \leq i e$ with $1 \leq i<p$ if $e$ is odd.

$L_{l, S}$ is maximal for $0 \leq l \leq e / 2$ and $e$ even. If $e$ is odd and $p=2$ or 3 then $K_{S}^{(r+p) \mathfrak{p}}=L_{(p-1) e, S}$ is optimal (cf. [36]). By comparison of conductors it is easily verified that $K_{S}^{(r+2) \mathfrak{p}}=K(y)$ where $y$ satisfies $y^{r}+y=x^{r+1}$ if $e$ is even, and $y^{q}-y=x^{r / p}\left(x^{q}-x\right)$ if $e$ is odd. For the connection with the Deligne-Lusztig curves associated with the Suzuki and the Ree group see [14] and [6].

For odd $e$, generalizing [29] one can write down defining equations for the first $p-1$ ray class fields.

3.5. Proposition. Let e be odd and let $x_{i}$ satisfy $x_{i}^{q}-x_{i}=x^{i r / p}\left(x^{q}-x\right)$. Then $K_{S}^{(r+i+1) \mathfrak{p}}=K\left(x_{1}, \ldots, x_{i}\right)$ for $1 \leq i<p$.

The proof is carried out in [1] using composites of Artin-Schreier extensions. There you also find defining equations for other ray class fields of the rational function field.

(B) General method. Now we develop a method to determine the numbers $\lambda_{S}^{(n)}$ for arbitrary ground field $K$ using ideas from Xing and Niederreiter [38] and thereby generalize what they call their "first construction".

We fix a basis $B$ of $\mathbb{F}_{q}$ over $\mathbb{F}_{p}$ and a uniformizer $\pi \in K_{\mathfrak{p}}^{*}$ at $\mathfrak{p}$. For each $j \in \mathbb{N}$ and $\beta \in B$ consider the cyclic subgroup

$$
G_{j \beta}^{(n)}:=\left\langle\left(1+\beta \pi^{j}\right) U^{(n)}\right\rangle
$$

of $U^{(1)} / U^{(n)}$. Its logarithmic order is

$$
\log _{p}\left|G_{j \beta}^{(n)}\right|=\left\lceil\begin{array}{c}
n \\
j
\end{array}\right\rceil:= \begin{cases}\left\lceil\log _{p}(n / j)\right\rceil & \text { if } j \leq n, \\
0 & \text { if } j \geq n .\end{cases}
$$

Denote by $\mathbb{N}^{*}:=\mathbb{N} \backslash \mathbb{Z} p$ the set of all positive integers not divisible by $p$. The numbers $\left\lceil\begin{array}{c}n \\ j\end{array}\right\rceil$ have the easily verified properties

$$
\begin{gathered}
{\left[\begin{array}{c}
n+1 \\
j
\end{array}\right]=\left[\begin{array}{c}
n \\
j
\end{array}\right]+ \begin{cases}1 & \text { if there is } l \in \mathbb{N}_{0} \text { such that } n=j p^{l} \\
0 & \text { otherwise },\end{cases} } \\
\sum_{j \in \mathbb{N}^{*}}\left\lceil\begin{array}{c}
n \\
j
\end{array}\right\rceil=n-1,
\end{gathered}
$$




$$
i, j \in \mathbb{N}, l \in \mathbb{Z}, i p^{l} \leq j \leq n \quad \Rightarrow \quad\left\lceil\begin{array}{c}
n \\
i
\end{array}\right\rceil \geq\left\lceil\begin{array}{c}
n \\
j
\end{array}\right\rceil+l .
$$

A straightforward proof using equation (6) yields the decomposition

$$
U^{(1)} / U^{(n)}=\prod_{\substack{j \in \mathbb{N}^{*} \\ \beta \in B}} G_{j \beta}^{(n)}
$$

as a direct product. Thus we obtain a non-canonical isomorphism of finite $p$-groups

$$
\begin{aligned}
\mu^{(n)}: U^{(1)} / U^{(n)} & \rightarrow \mathcal{M}^{(n)}:=\prod_{j \in \mathbb{N}^{*}}\left(\mathbb{Z} / \mathbb{Z} p^{\left.\Gamma_{j}^{n}\right\rceil}\right)^{B}, \\
\prod_{\substack{j \in \mathbb{N}^{*} \\
\beta \in B}}\left(1+\beta \pi^{j}\right)^{m_{j \beta}} U^{(n)} & \mapsto\left(m_{j \beta}+\mathbb{Z} p^{\left\lceil{ }_{j}^{n}\right\rceil}\right)_{\substack{j \in \mathbb{N}^{*} . \\
\beta \in B}}
\end{aligned}
$$

Passing to projective limits on both sides leads us to the isomorphism of free $\mathbb{Z}_{p}$-modules

$$
\mu: U^{(1)} \rightarrow \mathcal{M}:=\mathbb{Z}_{p}^{\mathbb{N}^{*} \times B},
$$

where as usual $\mathbb{Z}_{p} \subseteq \mathbb{Q}_{p}$ denotes the ring of $p$-adic integers (cf. Hasse's One-Unit Theorem in [10, p. 227]). Let ${ }^{(n)}: \mathcal{M} \rightarrow \mathcal{M}^{(n)}$ be the canonical projection. Then $\mu$ can be characterized as the unique map from $U^{(1)}$ to $\mathcal{M}$ satisfying $\mu(y)^{(n)}=\mu^{(n)}\left(y U^{(n)}\right)$ for all $y \in U^{(1)}$ and all $n$.

For $m=\left(m_{j \beta}\right)_{j \in \mathbb{N}^{*}, \beta \in B} \in \mathcal{M} \backslash\{0\}$ we put

$$
\nu(m):=\min \left\{j p^{v_{p}\left(m_{j \beta}\right)} \mid j \in \mathbb{N}^{*}, \beta \in B\right\} .
$$

Because for $j \in \mathbb{N}^{*}$ and $\beta \in B$ we have $m_{j \beta}^{(n)}=0$ iff $j p^{v_{p}\left(m_{j \beta}\right)} \geq n$, we can read $\nu(m)$ from $m^{(n)}$ as soon as $n>\nu(m)$. Let $i:=\nu(m)^{*}$ be the non-p-part of $\nu(m)$. Then there exists $\alpha \in B$ such that $i p^{v_{p}\left(m_{i \alpha}\right)}=\nu(m) \leq j p^{v_{p}\left(m_{j \beta}\right)}$ for all $j \in \mathbb{N}^{*}$ and $\beta \in B$. Using (8) we see that for each $n$ the logarithmic order of the cyclic group generated by $m^{(n)}$ is

$$
\begin{aligned}
\log _{p}\left|\mathbb{Z} m^{(n)}\right| & =\max \left\{0,\left\lceil\begin{array}{c}
n \\
j
\end{array}\right]-v_{p}\left(m_{j \beta}\right) \mid j \in \mathbb{N}^{*}, \beta \in B\right\} \\
& =\max \left\{0,\left[\begin{array}{c}
n \\
i
\end{array}\right]-v_{p}\left(m_{i \alpha}\right)\right\}=\left[\begin{array}{c}
n \\
\nu(m)
\end{array}\right] .
\end{aligned}
$$

Now let $r \in \mathbb{N}_{0}$ and $m_{i}=\left(m_{i j \beta}\right)_{j \in \mathbb{N}^{*}, \beta \in B} \in \mathcal{M}$ for $1 \leq i \leq r$ be given. In the following we describe a method to determine the order of the subgroup $\sum_{i=1}^{r} \mathbb{Z} m_{i}^{(n)} \subseteq \mathcal{M}^{(n)}$ for all $n \in \mathbb{N}$ simultaneously. We can assume without loss of generality that $m_{1}, \ldots, m_{r}$ are $\mathbb{Z}_{p}$-linearly independent and that $n_{1}:=\min \left\{\nu\left(m_{i}\right) \mid 1 \leq i \leq r\right\}=\nu\left(m_{1}\right)$. As above, for $j_{1}:=n_{1}^{*} \in \mathbb{N}^{*}$ there is $\beta_{1} \in B$ such that $v_{p}\left(m_{1 j_{1} \beta_{1}}\right)=v_{p}\left(n_{1}\right) \leq v_{p}\left(m_{i j_{1} \beta_{1}}\right)$ for all $i \in$ 
$\{1, \ldots, r\}$. Replacing $m_{i}$ by

we obtain

$$
\widetilde{m}_{i}:=m_{i}-\frac{m_{i j_{1} \beta_{1}}}{m_{1 j_{1} \beta_{1}}} m_{1} \quad \text { for } i=2, \ldots, r
$$

$$
\sum_{i=1}^{r} \mathbb{Z} m_{i}^{(n)}=\mathbb{Z} m_{1}^{(n)} \oplus \sum_{i=2}^{r} \mathbb{Z} \widetilde{m}_{i}^{(n)}
$$

and $\log _{p}\left|\mathbb{Z} m_{1}^{(n)}\right|=\left\lceil\begin{array}{c}n \\ n_{1}\end{array}\right\rceil$. We apply the same procedure to $\widetilde{m}_{2}, \ldots, \widetilde{m}_{r}$ and so on. In this way we obtain $n_{1} \leq \ldots \leq n_{r}$ such that

$$
\log _{p}\left|\sum_{i=1}^{r} \mathbb{Z} m_{i}^{(n)}\right|=\sum_{i=1}^{r}\left\lceil\begin{array}{c}
n \\
n_{i}
\end{array}\right\rceil \quad \forall n \in \mathbb{N} .
$$

As is evident from the construction, in order to determine $n_{1}, \ldots, n_{r}$ it suffices to perform the necessary linear transformations on $m_{1}^{(n)}, \ldots, m_{r}^{(n)}$ for an arbitrary $n>n_{r}$ rather than on $m_{1}, \ldots, m_{r}$.

Now suppose that we have found a basis $y_{1}, \ldots, y_{r}$ for the group $U_{S}$ with $r=|S|-1 \in \mathbb{N}_{0}$. Then by [12] we know that $y_{1}, \ldots, y_{r}$ are also $\mathbb{Z}_{p}$-linearly independent. Apply the described procedure to $m_{1}:=\mu\left(y_{1}\right), \ldots, m_{r}:=$ $\mu\left(y_{r}\right)$ to obtain $n_{1}, \ldots, n_{r}$ as above and define the polynomial

$$
\delta_{S}:=\sum_{i=1}^{r} t^{n_{i}} \in \mathbb{Z}[t] .
$$

Combining equations (6) and (9) with the exact sequence (3) results in the following recursive formula for the logarithmic orders $\lambda_{S}^{(n)}=\log _{p}\left(U^{(1)}\right.$ : $\left.U_{S} U^{(n)}\right)$, which at the same time shows that $\delta_{S}$ depends on $\mathfrak{p}$ and $S$ only.

3.6. Theorem. Write $\delta_{S}=\sum d_{n} t^{n}$ and let $\delta_{S}^{(n)}:=\sum_{l=0}^{v_{p}(n)} d_{n / p^{l}}=\mid\{i \mid$ $\left.1 \leq i \leq r, n_{i} \leq n, n_{i}^{*}=n^{*}\right\} \mid$. Then

$$
\lambda_{S}^{(n+1)}=\lambda_{S}^{(n)}+e-\delta_{S}^{(n)} \quad \text { for all } n \in \mathbb{N} .
$$

We call $\delta_{S}$ the $S$-description (at $\mathfrak{p}$ ) since together with $h_{S}$ it carries the complete information on the degrees and the genera of all ray class field extensions $K_{S}^{n \mathfrak{p}} \mid K$. While the above-mentioned "first construction" in [38] was restricted to the special case $q=p$ and $\delta_{S}=\sum_{j} t^{j}$ with the sum over the first $r$ elements of $\mathbb{N}^{*}$, we are now in a position to deal with practically any choice of $S$ and $\mathfrak{p}$.

3.7. EXAMPLE. We continue Example 2.3 and retain the notation therein. As we saw, the first $r \in\{1,2,3\}$ of the functions

$$
\begin{aligned}
& y_{1}=x^{13}(x+1)^{6} y^{-3}\left(y+x^{2}\right)^{-2} \\
& y_{2}=x^{5}(x+1)^{3} y^{-1}\left(y+x^{2}\right)^{-1} \\
& y_{3}=x^{-5}(x+1)^{-2} y\left(y+x^{2}\right)
\end{aligned}
$$


generate $U_{S_{r}}$. Taking $\mathfrak{p}=\mathfrak{p}_{0}$ and $\pi:=x$ as the uniformizer at $\mathfrak{p}$ yields the expansion $y=\pi^{3}+\pi^{5}+\pi^{6}+\pi^{10}+\ldots$, hence $\mu^{(6)}\left(y / \pi^{3}\right)=(2,1,1)$ and $\mu^{(6)}\left(\left(y+x^{2}\right) / \pi^{2}\right)=(1,1,0)$, where we write $\left(m_{1}, m_{3}, m_{5}\right)$ for short instead of $\left(m_{1}+8 \mathbb{Z}, m_{3}+2 \mathbb{Z}, m_{5}+2 \mathbb{Z}\right) \in \mathcal{M}^{(6)}$. We obtain the matrix

$$
\left(\begin{array}{l}
\mu^{(6)}\left(y_{1}\right) \\
\mu^{(6)}\left(y_{2}\right) \\
\mu^{(6)}\left(y_{3}\right)
\end{array}\right)=\left(\begin{array}{lll}
6 & 1 & 1 \\
0 & 0 & 1 \\
1 & 0 & 1
\end{array}\right)
$$

from which we read the descriptions $\delta_{S_{2}}=t^{2}+t^{5}$ and $\delta_{S_{3}}=t+t^{3}+t^{5}$. Theorem 3.6 yields

$$
\begin{aligned}
& \left(\lambda_{S_{2}}^{(n)}\right)_{n \in \mathbb{N}}=(0,1,1,2,2,2,3,4,4,5,5,6,7,8, \ldots), \\
& \left(\lambda_{S_{3}}^{(n)}\right)_{n \in \mathbb{N}}=(0,0,0,0,0,0,0,1,1,2, \ldots),
\end{aligned}
$$

so we see that $\mathfrak{p}_{3}$ does not split completely in $K_{S_{2}}^{n \mathfrak{p}}$ for $n \geq 2$, and formulas (4) and (5) lead to the values given in Table 3. By comparison with the Oesterlé bound we see that $L_{2, S_{2}}$ is optimal. Moreover compared to previous knowledge we have the improved estimates $25 \leq N_{2}(28) \leq 26$ and $49 \leq N_{2}(68) \leq 51$.

Table 3. Ray class fields of some $K \mid \mathbb{F}_{2}$ with $g(K)=2$

\begin{tabular}{lccccccccc}
\hline$l$ & 1 & 2 & 3 & 4 & 5 & 6 & 7 & 8 & 9 \\
$g\left(L_{l, S_{2}}\right)$ & 4 & 10 & 28 & 68 & 164 & 388 & 868 & 1892 & 4068 \\
$N\left(L_{l, S_{2}}\right)$ & 7 & 13 & 25 & 49 & 97 & 193 & 385 & 769 & 1537 \\
\hline
\end{tabular}

In the same manner we can calculate numerous examples. The essence of these is presented in the tables below.

Of course we can apply Method (B) to rational function fields as well, which is recommended where Method (A) fails. (Recall that Theorem 3.3 only ensures $\delta_{S}=\sum_{n} e_{S}^{(n)} t^{n}$ provided that $p n_{S}^{\prime} \geq n_{S}$.) E.g. for $q=16$ in addition to 25 possible descriptions which can be established by Theorem 3.3 we obtain 12 more using Method (B), namely $2 t+t^{2}, 2 t+t^{3}, 3 t+t^{2}, 3 t+t^{3}$, $3 t+2 t^{3}, 3 t+t^{2}+t^{3}, 3 t+t^{2}+2 t^{3}, 3 t+t^{2}+2 t^{3}+t^{7}, 4 t+3 t^{3}+t^{7}, 4 t+3 t^{3}+t^{5}+t^{7}$, $4 t+3 t^{3}+2 t^{5}+t^{7}$ and $4 t+3 t^{3}+2 t^{5}+2 t^{7}$.

Tables. Method (B) and the linear algebra necessary to compute the $e_{S}^{(n)}$ of Method (A) have been implemented in KASH, the KANT shell programming language (see [4] for details). I am grateful to the KANT group, and especially to F. Heß, for supporting me in my first steps with their extremely useful number theory tool.

Tables 4-9 below give the currently known range for the number $N_{q}(g)$ for some values of $q$ and $g$ in the form lower bound - upper bound, or a single 
value if $N_{q}(g)$ is known exactly. As a basis we use the results and tables of [9], [15], [17], [20], [24], [25], [26], [27], [28], [36], [38] and the estimates discussed in Section 2.

Each lower bound given is obtained by a corresponding field $L_{l, S}$ for an appropriate choice of $K, \mathfrak{p}, l$ and a set $S$ consisting of rational places only. We bold-face this lower bound wherever its previously known value is improved by our example. The entry for $n$ is chosen minimal with $l \leq \lambda_{S}^{(n)}$, i.e. $n=f\left(L_{l, S}, \mathfrak{p}\right)$ is the conductor exponent. Also note that we always have $N\left(L_{l, S}\right)=h_{S}\left(p^{l}|S|+\varepsilon\right)$ with $\varepsilon=0$ or 1 , which is guaranteed by explicitly verifying the supplementary condition to inequality (5).

Table 4. Ranges for $N_{2}(g)$

\begin{tabular}{rrrrrcc|ccccccc}
\hline$g$ & $N_{2}(g)$ & $n$ & $l$ & $|S|$ & $h_{S}$ & $g(K)$ & $g$ & $N_{2}(g)$ & $n$ & $l$ & $|S|$ & $h_{S}$ & $g(K)$ \\
\hline 6 & 10 & 2 & 1 & 1 & 5 & 1 & 55 & $\mathbf{4 1 - 4 3}$ & 12 & 3 & 5 & 1 & 3 \\
8 & 11 & 10 & 1 & 5 & 1 & 2 & 58 & $\mathbf{4 1}-45$ & 12 & 3 & 5 & 1 & 4 \\
10 & 13 & 4 & 2 & 3 & 1 & 2 & 60 & $\mathbf{4 1}-47$ & 11 & 3 & 5 & 1 & 4 \\
13 & 15 & 12 & 1 & 7 & 1 & 4 & 61 & $\mathbf{4 1}-47$ & 14 & 3 & 5 & 1 & 3 \\
14 & $15-16$ & 14 & 1 & 7 & 1 & 4 & 68 & $\mathbf{4 9}-51$ & 8 & 4 & 3 & 1 & 2 \\
15 & 17 & 7 & 3 & 2 & 1 & 0 & 69 & $49-52$ & 16 & 3 & 6 & 1 & 3 \\
16 & $\mathbf{1 7}-18$ & 14 & 1 & 8 & 1 & 5 & 71 & $\mathbf{4 9}-53$ & 6 & 4 & 3 & 1 & 3 \\
17 & $17-18$ & 5 & 4 & 1 & 1 & 0 & 74 & $\mathbf{4 9}-55$ & 16 & 3 & 6 & 1 & 4 \\
20 & $19-21$ & 0 & 0 & 1 & 19 & 2 & 75 & $\mathbf{4 9}-56$ & 14 & 3 & 6 & 1 & 5 \\
22 & $21-22$ & 12 & 2 & 5 & 1 & 2 & 79 & $52-58$ & 0 & 0 & 2 & 26 & 4 \\
28 & $\mathbf{2 5}-26$ & 7 & 3 & 3 & 1 & 2 & 81 & $\mathbf{4 9}-59$ & 15 & 3 & 6 & 1 & 5 \\
29 & $25-27$ & 14 & 2 & 6 & 1 & 3 & 83 & $\mathbf{5 7}-60$ & 18 & 3 & 7 & 1 & 4 \\
30 & $\mathbf{2 5}-27$ & 12 & 2 & 6 & 1 & 4 & 84 & $57-61$ & 18 & 3 & 7 & 1 & 4 \\
35 & $\mathbf{2 9}-31$ & 16 & 2 & 7 & 1 & 4 & 89 & $\mathbf{5 7}-64$ & 18 & 3 & 7 & 1 & 5 \\
37 & $\mathbf{2 9}-32$ & 14 & 2 & 7 & 1 & 5 & 91 & $\mathbf{5 7}-65$ & 18 & 3 & 7 & 1 & 5 \\
39 & 33 & 8 & 4 & 2 & 1 & 0 & 95 & $65-68$ & 14 & 4 & 4 & 1 & 1 \\
41 & $\mathbf{3 3}-35$ & 6 & 3 & 4 & 1 & 4 & 97 & $\mathbf{6 5}-69$ & 8 & 4 & 4 & 1 & 4 \\
42 & $\mathbf{3 3}-35$ & 18 & 2 & 8 & 1 & 5 & 98 & $\mathbf{6 5}-69$ & 20 & 3 & 8 & 1 & 5 \\
44 & $\mathbf{3 3}-37$ & 11 & 3 & 4 & 1 & 2 & 100 & $\mathbf{6 5}-70$ & 12 & 4 & 4 & 1 & 2 \\
\hline
\end{tabular}

Table 5. Ranges for $N_{3}(g)$

\begin{tabular}{rrrcccc|ccccccc}
\hline$g$ & $N_{3}(g)$ & $n$ & $l$ & $|S|$ & $h_{S}$ & $g(K)$ & $g$ & $N_{3}(g)$ & $n$ & $l$ & $|S|$ & $h_{S}$ & $g(K)$ \\
\hline 5 & $12-13$ & 0 & 0 & 6 & 2 & 3 & 24 & $\mathbf{3 1}-38$ & 14 & 1 & 10 & 1 & 4 \\
7 & $16-17$ & 3 & 1 & 5 & 1 & 2 & 30 & $37-46$ & 8 & 2 & 4 & 1 & 1 \\
9 & 19 & 5 & 1 & 6 & 1 & 2 & 33 & $\mathbf{4 6}-49$ & 4 & 2 & 5 & 1 & 3 \\
10 & $19-21$ & 5 & 2 & 2 & 1 & 0 & 36 & $46-52$ & 9 & 2 & 5 & 1 & 1 \\
13 & $24-25$ & 0 & 0 & 2 & 12 & 2 & 43 & $55-60$ & 11 & 2 & 6 & 1 & 1 \\
15 & 28 & 6 & 2 & 3 & 1 & 0 & 46 & $55-63$ & 6 & 3 & 2 & 1 & 0 \\
17 & $24-30$ & 5 & 1 & 4 & 2 & 2 & 47 & $\mathbf{5 4}-65$ & 6 & 2 & 3 & 2 & 1 \\
19 & $28-32$ & 12 & 1 & 9 & 1 & 3 & 48 & $55-66$ & 11 & 2 & 6 & 1 & 2 \\
\hline
\end{tabular}


Table 5 (cont.)

\begin{tabular}{ccccccc|ccccccc}
\hline$g$ & $N_{3}(g)$ & $n$ & $l$ & $|S|$ & $h_{S}$ & $g(K)$ & $g$ & $N_{3}(g)$ & $n$ & $l$ & $|S|$ & $h_{S}$ & $g(K)$ \\
\hline 52 & $\mathbf{5 5}-70$ & 11 & 2 & 6 & 1 & 2 & 73 & $\mathbf{8 2}-92$ & 14 & 2 & 9 & 1 & 3 \\
54 & $\mathbf{5 5}-72$ & 9 & 2 & 6 & 1 & 3 & 75 & $\mathbf{7 3}-95$ & 12 & 2 & 8 & 1 & 4 \\
55 & $\mathbf{6 4}-73$ & 12 & 2 & 7 & 1 & 2 & 78 & $\mathbf{7 3}-98$ & 14 & 2 & 8 & 1 & 4 \\
57 & $\mathbf{7 0}-75$ & 2 & 1 & 3 & 7 & 3 & 79 & $\mathbf{7 3}-99$ & 14 & 2 & 8 & 1 & 4 \\
58 & $\mathbf{5 5}-77$ & 11 & 2 & 6 & 1 & 3 & 81 & $\mathbf{8 2}-101$ & 14 & 2 & 9 & 1 & 4 \\
60 & $\mathbf{6 4}-79$ & 11 & 2 & 7 & 1 & 3 & 82 & $\mathbf{7 3}-102$ & 14 & 2 & 8 & 1 & 4 \\
63 & $\mathbf{6 4}-82$ & 12 & 2 & 7 & 1 & 3 & 84 & $\mathbf{8 2}-104$ & 15 & 2 & 9 & 1 & 4 \\
64 & $\mathbf{6 4}-83$ & 12 & 2 & 7 & 1 & 3 & 85 & $\mathbf{8 4}-105$ & 6 & 1 & 4 & 7 & 3 \\
65 & $\mathbf{7 2}-84$ & 6 & 2 & 4 & 2 & 2 & 87 & $\mathbf{8 2}-107$ & 5 & 3 & 3 & 1 & 2 \\
66 & $\mathbf{6 4}-85$ & 12 & 2 & 7 & 1 & 3 & 93 & $\mathbf{9 1}-113$ & 17 & 2 & 10 & 1 & 4 \\
69 & $82-88$ & 8 & 3 & 3 & 1 & 0 & 94 & $\mathbf{9 1}-114$ & 17 & 2 & 10 & 1 & 4 \\
70 & $\mathbf{6 4}-89$ & 11 & 2 & 7 & 1 & 4 & 96 & $\mathbf{8 2}-116$ & 8 & 3 & 3 & 1 & 1 \\
72 & $\mathbf{6 4}-91$ & 12 & 2 & 7 & 1 & 4 & 99 & $\mathbf{1 0 0}-119$ & 18 & 2 & 11 & 1 & 4 \\
\hline
\end{tabular}

Table 6. Ranges for $N_{4}(g)$

\begin{tabular}{rrrllll|rrrrrrr}
\hline$g$ & $N_{4}(g)$ & $n$ & $l$ & $|S|$ & $h_{S}$ & $g(K)$ & $g$ & $N_{4}(g)$ & $n$ & $l$ & $|S|$ & $h_{S}$ & $g(K)$ \\
\hline 3 & 14 & 2 & 1 & 3 & 2 & 1 & 61 & $99-108$ & 4 & 3 & 4 & 3 & 2 \\
4 & 15 & 6 & 1 & 7 & 1 & 1 & 62 & $\mathbf{9 7}-109$ & 7 & 4 & 6 & 1 & 2 \\
5 & $17-18$ & 6 & 2 & 4 & 1 & 0 & 63 & $\mathbf{8 9}-111$ & 14 & 3 & 11 & 1 & 3 \\
6 & 20 & 0 & 0 & 4 & 5 & 2 & 64 & $\mathbf{9 9}-112$ & 3 & 4 & 2 & 3 & 1 \\
8 & $21-24$ & 6 & 1 & 10 & 1 & 3 & 65 & $98-114$ & 5 & 4 & 3 & 2 & 1 \\
9 & 26 & 3 & 2 & 3 & 2 & 1 & 66 & $\mathbf{9 7}-115$ & 10 & 4 & 6 & 1 & 1 \\
10 & $27-28$ & 2 & 2 & 2 & 3 & 1 & 67 & $\mathbf{9 7}-117$ & 7 & 5 & 3 & 1 & 0 \\
12 & $\mathbf{2 9}-31$ & 8 & 2 & 7 & 1 & 1 & 68 & $\mathbf{9 7}-118$ & 10 & 4 & 6 & 1 & 1 \\
13 & 33 & 6 & 3 & 4 & 1 & 0 & 71 & $\mathbf{9 7}-123$ & 8 & 4 & 6 & 1 & 2 \\
19 & $\mathbf{3 7}-43$ & 10 & 2 & 9 & 1 & 2 & 72 & $\mathbf{1 1 3}-124$ & 10 & 4 & 7 & 1 & 1 \\
20 & $\mathbf{3 7}-45$ & 10 & 2 & 9 & 1 & 2 & 73 & $\mathbf{1 1 3}-125$ & 10 & 4 & 7 & 1 & 1 \\
21 & $41-47$ & 8 & 2 & 10 & 1 & 3 & 74 & $\mathbf{9 7}-127$ & 8 & 4 & 6 & 1 & 2 \\
22 & $\mathbf{4 1 - 4 8}$ & 10 & 2 & 10 & 1 & 3 & 76 & $99-130$ & 3 & 3 & 4 & 3 & 3 \\
23 & $\mathbf{4 1}-50$ & 7 & 3 & 5 & 1 & 1 & 78 & $\mathbf{9 7}-133$ & 7 & 4 & 6 & 1 & 3 \\
25 & $51-53$ & 3 & 2 & 4 & 3 & 2 & 80 & $\mathbf{1 1 3}-135$ & 10 & 4 & 7 & 1 & 2 \\
27 & $49-56$ & 6 & 4 & 3 & 1 & 0 & 81 & $129-137$ & 8 & 5 & 4 & 1 & 0 \\
28 & $\mathbf{5 1}-58$ & 3 & 3 & 2 & 3 & 1 & 82 & $\mathbf{1 1 3}-138$ & 10 & 4 & 7 & 1 & 2 \\
32 & $\mathbf{5 7}-65$ & 10 & 3 & 7 & 1 & 1 & 83 & $\mathbf{1 1 3}-140$ & 10 & 4 & 7 & 1 & 2 \\
33 & $65-66$ & 7 & 4 & 4 & 1 & 0 & 84 & $\mathbf{1 1 3}-141$ & 10 & 4 & 7 & 1 & 2 \\
34 & $57-68$ & 8 & 3 & 7 & 1 & 2 & 86 & $\mathbf{1 1 3}-144$ & 10 & 4 & 7 & 1 & 2 \\
41 & $65-78$ & 10 & 3 & 8 & 1 & 2 & 89 & $\mathbf{1 1 3}-148$ & 10 & 4 & 7 & 1 & 2 \\
47 & $\mathbf{7 3}-87$ & 12 & 3 & 9 & 1 & 2 & 94 & $129-155$ & 11 & 4 & 8 & 1 & 2 \\
52 & $\mathbf{8 1}-95$ & 11 & 3 & 10 & 1 & 3 & 95 & $\mathbf{1 1 3}-156$ & 10 & 4 & 7 & 1 & 3 \\
53 & $\mathbf{8 1}-96$ & 12 & 3 & 10 & 1 & 3 & 97 & $\mathbf{1 2 9}-159$ & 12 & 4 & 8 & 1 & 2 \\
55 & $\mathbf{8 1 - 9 9}$ & 8 & 4 & 5 & 1 & 1 & 98 & $\mathbf{1 2 9}-161$ & 7 & 5 & 4 & 1 & 1 \\
56 & $\mathbf{8 1}-101$ & 12 & 3 & 10 & 1 & 3 & 99 & $\mathbf{1 2 9}-162$ & 12 & 4 & 8 & 1 & 2 \\
57 & $\mathbf{8 1}-102$ & 6 & 4 & 5 & 1 & 2 & 100 & $\mathbf{1 2 9}-163$ & 10 & 4 & 8 & 1 & 3 \\
58 & $\mathbf{9 7}-103$ & 8 & 4 & 6 & 1 & 1 & & & & & & \\
\hline & & & & & & & & & & & &
\end{tabular}


Table 7. Ranges for $N_{q}(g)$ with $q=5,7,8$ and 9

\begin{tabular}{rrrrr}
\hline$g$ & $N_{5}(g)$ & $n$ & $l$ & $|S|$ \\
\hline 6 & $21-25$ & 5 & 1 & 4 \\
22 & $51-60$ & 4 & 2 & 2 \\
34 & $76-83$ & 5 & 2 & 3 \\
56 & $101-125$ & 7 & 2 & 4 \\
70 & $126-150$ & 8 & 2 & 5 \\
\hline & & & & \\
\hline$g$ & $N_{7}(g)$ & $n$ & $l$ & $|S|$ \\
\hline 6 & $\mathbf{2 2 - 3 2}$ & 4 & 1 & 3 \\
9 & $\mathbf{2 9 - 4 2}$ & 5 & 1 & 4 \\
12 & $\mathbf{3 6}-52$ & 6 & 1 & 5 \\
15 & $\mathbf{4 3 - 6 0}$ & 7 & 1 & 6 \\
45 & $\mathbf{9 9}-140$ & 4 & 2 & 2 \\
69 & $\mathbf{1 4 8}-198$ & 5 & 2 & 3 \\
93 & $\mathbf{1 9 7}-254$ & 6 & 2 & 4 \\
\hline
\end{tabular}

\begin{tabular}{rrrrr}
\hline$g$ & \multicolumn{1}{c}{$N_{8}(g)$} & $n$ & $l$ & $|S|$ \\
\hline 6 & $33-36$ & 6 & 2 & 8 \\
7 & $33-39$ & 4 & 3 & 4 \\
14 & 65 & 6 & 3 & 8 \\
29 & $97-109$ & 6 & 4 & 6 \\
38 & $129-135$ & 8 & 4 & 8 \\
46 & $129-158$ & 6 & 5 & 4 \\
\hline & & & & \\
\hline$g$ & $N_{9}(g)$ & $n$ & $l$ & $|S|$ \\
\hline 1 & 16 & 3 & 1 & 5 \\
3 & 28 & 5 & 1 & 9 \\
12 & $55-63$ & 5 & 2 & 6 \\
15 & $64-74$ & 6 & 2 & 7 \\
21 & $82-95$ & 8 & 2 & 9 \\
48 & $163-180$ & 6 & 3 & 6 \\
\hline
\end{tabular}

Table 8. Ranges for $N_{q}(g)$ with $q=16,25,27$ and 49

\begin{tabular}{rrccc}
\hline$g$ & $N_{16}(g)$ & $n$ & $l$ & $|S|$ \\
\hline 1 & 25 & 4 & 1 & 12 \\
2 & 33 & 6 & 1 & 16 \\
5 & $49-55$ & 6 & 2 & 12 \\
6 & 65 & 6 & 2 & 16 \\
13 & $97-103$ & 6 & 3 & 12 \\
14 & $97-108$ & 6 & 3 & 12 \\
18 & $113-129$ & 8 & 3 & 14 \\
22 & $129-150$ & 10 & 3 & 16 \\
27 & $\mathbf{1 4 5}-176$ & 6 & 4 & 9 \\
29 & $161-187$ & 6 & 4 & 10 \\
30 & $161-192$ & 6 & 4 & 10 \\
33 & $\mathbf{1 9 3}-207$ & 7 & 4 & 12 \\
38 & $\mathbf{1 9 3}-233$ & 8 & 4 & 12 \\
42 & $\mathbf{2 0 9}-254$ & 8 & 4 & 13 \\
50 & $\mathbf{2 2 5}-291$ & 10 & 4 & 14 \\
\hline & & & & \\
\hline$g$ & $N_{49}(g)$ & $n$ & $l$ & $|S|$ \\
\hline 3 & 92 & 3 & 1 & 13 \\
9 & 176 & 5 & 1 & 25 \\
21 & 344 & 9 & 1 & 49 \\
45 & $\mathbf{4 4 2 - 5 7 9}$ & 4 & 2 & 9 \\
\hline & & &
\end{tabular}

\begin{tabular}{rrrcc}
\hline$g$ & $N_{25}(g)$ & $n$ & $l$ & $|S|$ \\
\hline 2 & 46 & 3 & 1 & 9 \\
4 & 66 & 4 & 1 & 13 \\
6 & $\mathbf{6 6}-85$ & 5 & 1 & 13 \\
10 & 126 & 7 & 1 & 25 \\
12 & $\mathbf{1 0 1}-140$ & 3 & 2 & 4 \\
22 & $\mathbf{1 7 6}-208$ & 4 & 2 & 7 \\
24 & $\mathbf{1 7 6}-222$ & 4 & 2 & 7 \\
32 & $\mathbf{2 2 6}-275$ & 5 & 2 & 9 \\
34 & $\mathbf{2 2 6}-289$ & 5 & 2 & 9 \\
36 & $\mathbf{2 2 6}-302$ & 5 & 2 & 9 \\
\hline & & & & \\
\hline$g$ & $N_{27}(g)$ & $n$ & $l$ & $|S|$ \\
\hline 4 & $64-68$ & 3 & 2 & 7 \\
10 & $\mathbf{9 1}-128$ & 5 & 2 & 10 \\
12 & $109-148$ & 5 & 2 & 12 \\
13 & $136-156$ & 3 & 3 & 5 \\
15 & $\mathbf{1 3 6}-171$ & 6 & 2 & 15 \\
16 & $\mathbf{1 3 6}-178$ & 6 & 2 & 15 \\
21 & $163-214$ & 8 & 2 & 18 \\
36 & $244-319$ & 11 & 2 & 27 \\
39 & $271-340$ & 5 & 3 & 10 \\
48 & $\mathbf{3 2 5}-402$ & 6 & 3 & 12 \\
\hline
\end{tabular}


Table 9. Ranges for $N_{q}(g)$ with $q=32,64$ and 81

\begin{tabular}{rrccc}
\hline$g$ & \multicolumn{1}{c}{$N_{32}(g)$} & $n$ & $l$ & $|S|$ \\
\hline 6 & $81-99$ & 6 & 2 & 20 \\
11 & $\mathbf{1 1 3}-154$ & 6 & 3 & 14 \\
12 & $129-165$ & 10 & 2 & 32 \\
13 & $\mathbf{1 2 9}-176$ & 6 & 3 & 16 \\
14 & $145-187$ & 6 & 3 & 18 \\
18 & $\mathbf{1 6 1}-220$ & 8 & 3 & 20 \\
27 & $\mathbf{2 0 9}-290$ & 10 & 3 & 26 \\
28 & $257-298$ & 10 & 3 & 32 \\
29 & $\mathbf{2 2 5}-306$ & 6 & 4 & 14 \\
30 & $273-313$ & 6 & 4 & 17 \\
38 & $\mathbf{2 8 9}-375$ & 8 & 4 & 18 \\
\hline & & & & \\
\hline$g$ & $N_{81}(g)$ & $n$ & $l$ & $|S|$ \\
\hline 1 & 100 & 3 & 1 & 33 \\
3 & 136 & 5 & 1 & 45 \\
4 & 154 & 6 & 1 & 51 \\
9 & 244 & 11 & 1 & 81 \\
12 & 298 & 5 & 2 & 33 \\
15 & $\mathbf{2 8 0}-352$ & 6 & 2 & 31 \\
21 & $\mathbf{3 5 2}-459$ & 8 & 2 & 39 \\
31 & $\mathbf{4 6 0}-639$ & 11 & 2 & 51 \\
36 & 730 & 11 & 2 & 81 \\
48 & $\mathbf{6 7 6}-885$ & 6 & 3 & 25 \\
\hline & & & &
\end{tabular}

\begin{tabular}{rrccc}
\hline$g$ & $N_{64}(g)$ & $n$ & $l$ & $|S|$ \\
\hline 1 & 81 & 4 & 1 & 40 \\
2 & 97 & 6 & 1 & 48 \\
4 & 129 & 10 & 1 & 64 \\
6 & 161 & 6 & 2 & 40 \\
8 & $\mathbf{1 6 9}-193$ & 8 & 2 & 42 \\
11 & $201-241$ & 10 & 2 & 50 \\
12 & 257 & 10 & 2 & 64 \\
13 & $\mathbf{2 2 5}-272$ & 6 & 3 & 28 \\
14 & $\mathbf{2 4 1}-288$ & 6 & 3 & 30 \\
18 & $\mathbf{2 8 1}-352$ & 8 & 3 & 35 \\
22 & $\mathbf{3 2 1}-416$ & 10 & 3 & 40 \\
24 & $\mathbf{3 3 7}-448$ & 10 & 3 & 42 \\
26 & $\mathbf{3 8 5}-480$ & 10 & 3 & 48 \\
27 & $401-496$ & 10 & 3 & 50 \\
28 & 513 & 10 & 3 & 64 \\
30 & $\mathbf{3 8 5}-536$ & 6 & 4 & 24 \\
38 & $\mathbf{4 4 9}-627$ & 8 & 4 & 28 \\
50 & $\mathbf{5 6 1}-762$ & 10 & 4 & 35 \\
\hline
\end{tabular}

All examples for $q \geq 5$ arise from the rational function field and are usually computed by means of the quicker Method (A). For $q=2,3$ and 4, where we have also considered ray class fields of fields other than the rational function field, we add two more columns to the table, one for the $S$-class number $h_{S}$ and one for the genus $g(K)$ of the ground field $K$ used, which in most cases is borrowed from [18] and [19]. More detailed tables giving precise reference to how each example using Method (B) is obtained are found in [1].

Appendix: Ramification groups. Let $L \mid K$ be a finite Galois extension of global (or local) fields with group $G(L \mid K), \mathfrak{p}$ a (non-archimedian) place of $K$ and $\mathfrak{q}$ a place of $L$ lying above $\mathfrak{p}$. For $s \in[-1, \infty)$ we have the lower ramification groups of $\mathfrak{q} \mid \mathfrak{p}$,

$$
G_{s}(\mathfrak{q} \mid \mathfrak{p}):=\left\{\sigma \in G(L \mid K) \mid v_{\mathfrak{q}}(\sigma z-z) \geq s+1 \text { for all } z \in L \text { integral at } \mathfrak{q}\right\},
$$

which are renumbered to define the upper ramification groups $G^{\eta(s)}(\mathfrak{q} \mid \mathfrak{p}):=$ $G_{s}(\mathfrak{q} \mid \mathfrak{p})$ by means of the continuous bijection

$$
\eta:[-1, \infty) \rightarrow[-1, \infty), \quad s \mapsto \int_{0}^{s} \frac{\left|G_{x}(\mathfrak{q} \mid \mathfrak{p})\right|}{\left|G_{0}(\mathfrak{q} \mid \mathfrak{p})\right|} d x
$$


(cf. [16, pp. 186ff]). Note that $\eta$ is the identity at least on $[-1,0]$ and that $G_{-1}(\mathfrak{q} \mid \mathfrak{p})=G^{-1}(\mathfrak{q} \mid \mathfrak{p})$ is the decomposition group and $G_{0}(\mathfrak{q} \mid \mathfrak{p})=G^{0}(\mathfrak{q} \mid \mathfrak{p})$ is the inertia group of $\mathfrak{q} \mid \mathfrak{p}$.

Now let $L \mid K$ be abelian. Then the ramification groups do no longer depend on the specific choice of $\mathfrak{q}$, so we can write $G^{s}(L, \mathfrak{p})$ instead of $G^{s}(\mathfrak{q} \mid \mathfrak{p})$. In Section 1 we made use of an upper index version of Hilbert's Then

Different Formula. Denote by $d(\mathfrak{q} \mid \mathfrak{p})$ the different exponent of $\mathfrak{q} \mid \mathfrak{p}$.

$$
d(\mathfrak{q} \mid \mathfrak{p})=\sum_{n=0}^{\infty}\left(\left|G^{0}(L, \mathfrak{p})\right|-\left(G^{0}(L, \mathfrak{p}): G^{n}(L, \mathfrak{p})\right)\right) .
$$

Proof. For convenience put $G^{s}:=G^{s}(L, \mathfrak{p})$ and $\psi:=\eta^{-1}$, where $\eta$ is defined as above. Let $t_{0}:=-1$, and let $0 \leq t_{1}<\ldots<t_{r}$ be the (other) jumps of the filtration $\left(G^{s}\right)_{s \geq-1}$, i.e. the numbers $s \geq-1$ satisfying $G^{s+\varepsilon} \subsetneq G^{s}$ for all $\varepsilon>0$ (cf. [16, p. 372]). Obviously all $\psi\left(t_{i}\right)$ are integers, and $t_{0}=-1$ is a jump iff the inertia degree of $\mathfrak{q} \mid \mathfrak{p}$ is $>1$. The bijection $\eta$ is piecewise linear, its slopes being

$$
\eta^{\prime}(s)=\frac{1}{\left(G^{0}: G^{t_{i}}\right)}=\frac{t_{i}-t_{i-1}}{\psi\left(t_{i}\right)-\psi\left(t_{i-1}\right)}
$$

for $\psi\left(t_{i-1}\right)<s<\psi\left(t_{i}\right)$ and $i \in\{1, \ldots, r\}$. By the Hasse-Arf theorem the $t_{i}$ are integers, too. Substituting into Hilbert's (lower index) different formula (see [37, p. 124]) yields

$$
\begin{aligned}
d(\mathfrak{q} \mid \mathfrak{p}) & =\sum_{n=0}^{\infty}\left(\left|G_{n}(\mathfrak{q} \mid \mathfrak{p})\right|-1\right)=\sum_{i=1}^{r} \sum_{s=\psi\left(t_{i-1}\right)+1}^{\psi\left(t_{i}\right)}\left(\left|G^{t_{i}}\right|-1\right) \\
& =\sum_{i=1}^{r}\left(\psi\left(t_{i}\right)-\psi\left(t_{i-1}\right)\right) \frac{\left|G^{0}\right|-\left(G^{0}: G^{t_{i}}\right)}{\left(G^{0}: G^{t_{i}}\right)} \\
& =\sum_{i=1}^{r}\left(t_{i}-t_{i-1}\right)\left(\left|G^{0}\right|-\left(G^{0}: G^{t_{i}}\right)\right) \\
& =\sum_{i=1}^{r} \sum_{n=t_{i-1}+1}^{t_{i}}\left(\left|G^{0}\right|-\left(G^{0}: G^{n}\right)\right)=\sum_{n=0}^{\infty}\left(\left|G^{0}\right|-\left(G^{0}: G^{n}\right)\right) .
\end{aligned}
$$

\section{References}

[1] R. Auer, Ray class fields of global function fields with many rational places, Dissertation at the University of Oldenburg, www.bis.uni-oldenburg.de/dissertation/ ediss.html, 1999.

[2] J. W. S. Cassels and A. Fröhlich, Algebraic Number Theory, Academic Press, New York, 1967. 
[3] H. Cohen, F. Diaz y Diaz and M. Olivier, Computing ray class groups, conductors and discriminants, in: Algorithmic Number Theory, H. Cohen (ed.), Lecture Notes in Comput. Sci. 1122, Springer, 1996, 49-57.

[4] M. Daberkow, C. Fieker, J. Klüners, M. Pohst, K. Roegner, M. Schörnig and K. Wildanger, KANT V4, J. Symbolic Comput. 24 (1997), 267-283.

[5] R. Fuhrmann and F. Torres, The genus of curves over finite fields with many rational points, Manuscripta Math. 89 (1996), 103-106.

[6] A. Garcia and H. Stichtenoth, Algebraic function fields over finite fields with many rational places, IEEE Trans. Inform. Theory 41 (1995), 1548-1563.

[7] G. van der Geer and M. van der Vlugt, How to construct curves over finite fields with many points, in: Arithmetic Geometry (Cortona, 1984), F. Catanese (ed.), Cambridge Univ. Press, 1997, 169-189.

[8] - - - Constructing curves over finite fields with many points by solving linear equations, preprint, 1997.

[9] —, 一, Tables of curves with many points, preprint at http://www.wins.uva.nl/ ${ }^{\sim}$ geer, 1999.

[10] H. Hasse, Number Theory, Springer, Berlin, 1980.

[11] D. R. Hayes, Explicit class field theory in global function fields, in: Studies in Algebra and Number Theory, Adv. in Math. Suppl. Stud. 6, Academic Press, 1979, $173-217$.

[12] H. Kisilevsky, Multiplicative independence in function fields, J. Number Theory 44 (1993), 352-355.

[13] K. Lauter, Ray class field constructions of curves over finite fields with many rational points, in: Algorithmic Number Theory, H. Cohen (ed.), Lecture Notes in Comput. Sci. 1122, Springer, 1996, 187-195.

[14] -, Deligne-Lusztig curves as ray class fields, Manuscripta Math. 98 (1999), 87-96.

[15] - A formula for constructing curves over finite fields with many rational points, J. Number Theory 74 (1999), 56-72.

[16] J. Neukirch, Algebraische Zahlentheorie, Springer, Berlin, 1991.

[17] H. Niederreiter, Nets, $(t, s)$-sequences, and algebraic curves over finite fields with many rational points, in: Proc. Internat. Congress of Math. (Berlin, 1998), Documenta Math. Extra Vol. ICM III (1998), 377-386.

[18] H. Niederreiter and C. P. Xing, Explicit global function fields over the binary field with many rational places, Acta Arith. 75 (1996), 383-396.

[19] -, -, Cyclotomic function fields, Hilbert class fields, and global function fields with many rational places, ibid. 79 (1997), 59-76.

[20] - - - Drinfeld modules of rank 1 and algebraic curves with many rational points. II, ibid. 81 (1997), 81-100.

[21] —, - Algebraic curves over finite fields with many rational points, in: Proc. Number Theory Conf. (Eger, 1996), de Gruyter, 1998, 423-443.

[22] —, - Global function fields with many rational places over the ternary field, Acta Arith. 83 (1998), 65-86.

[23] - - - Global function fields with many rational places over the quinary field, Demonstratio Math. 30 (1997), 919-930.

[24] - - - Global function fields with many rational places over the quinary field. II, Acta Arith. 86 (1998), 277-288.

[25] - - - Algebraic curves with many rational points over finite fields of characteristic 2, in: Number Theory in Progress (Zakopane, 1997), Vol. 1, de Gruyter, 1999, $359-380$. 
[26] H. Niederreiter and C. P. Xing, A general method of constructing global function fields with many rational places, in: Algorithmic Number Theory (Portland, 1998), Lecture Notes in Comput. Sci. 1423, Springer, 1998, 555-566.

[27] - - - Algebraic curves over finite fields with many rational points and their applications, in: Number Theory, V. C. Dumir et al. (eds.), Indian National Science Academy, to appear.

[28] - - - Global function fields with many rational places and their applications, Contemp. Math. 225 (1999), 87-111.

[29] J. P. Pedersen, A function field related to the Ree group, in: Coding Theory and Algebraic Geometry (Luminy, 1991), H. Stichtenoth and M. A. Tsfasman (eds.), Lecture Notes in Math. 1518, Springer, Berlin, 1992, 122-131.

[30] M. Perret, Tours ramifiées infinies de corps de classes, J. Number Theory 38 (1991), 300-322.

[31] M. Rosen, $S$-units and $S$-class group in algebraic function fields, J. Algebra 26 (1973), 98-108.

[32] —, The Hilbert class field in function fields, Exposition. Math. 5 (1987), 365-378.

[33] R. Schoof, Algebraic Curves and Coding Theory, UTM 336 (1990), Univ. of Trento.

[34] J.-P. Serre, Sur le nombre des points rationnelles d'une courbe algébrique sur un corps fini, C. R. Acad. Sci. Paris Sér. I 296 (1983), 397-402.

[35] -, Nombres de points des courbes algébrique sur $\mathbb{F}_{q}$, Sém. Théor. Nombres Bordeaux $22(1982 / 83)$.

[36] —, Résumé des cours de 1983-1984, Annuaire du Collège de France, 1984, 79-83.

[37] H. Stichtenoth, Algebraic Function Fields and Codes, Springer, Berlin, 1993.

[38] C. P. Xing and H. Niederreiter, Drinfel'd modules of rank 1 and algebraic curves with many rational points, Monatsh. Math. 127 (1999), 219-241.

Afd. wiskunde

Rijksuniversiteit Groningen

Blauwborgje 3

NL-9747 AC Groningen, The Netherlands

E-mail: auer@math.rug.nl 